Pacific

Journal of

Mathematics

THE BOREL-WEIL THEOREM FOR REDUCTIVE LIE GROUPS

José ARAujo AND Tim BRATTEN 


\title{
THE BOREL-WEIL THEOREM FOR REDUCTIVE LIE GROUPS
}

\author{
José ARAujo AND TIM BRATTEN
}

\begin{abstract}
In this manuscript we consider the extent to which an irreducible representation for a reductive Lie group can be realized as the sheaf cohomology of an equivariant holomorphic line bundle defined on an open invariant submanifold of a complex flag space. Our main result is the following: suppose $G_{0}$ is a real reductive group of Harish-Chandra class and let $X$ be the associated full complex flag space. Suppose $\mathcal{O}_{\lambda}$ is the sheaf of sections of a $\boldsymbol{G}_{0}$ equivariant holomorphic line bundle on $X$ whose parameter $\lambda$ (in the usual twisted $\mathcal{D}$-module context) is antidominant and regular. Let $S \subseteq X$ be a $G_{0}$-orbit and suppose $U \supseteq S$ is the smallest $G_{0}$-invariant open submanifold of $X$ that contains $S$. From the analytic localization theory of Hecht and Taylor one knows that there is a nonnegative integer $q$ such that the compactly supported sheaf cohomology groups $H_{\mathrm{c}}{ }^{p}\left(S, \mathcal{O}_{\lambda} \mid s\right)$ vanish except in degree $q$, in which case $H_{\mathrm{c}}^{q}\left(S, \mathcal{O}_{\lambda} \mid s\right)$ is the minimal globalization of an associated standard Beilinson-Bernstein module. In this study, we show that the $q$-th compactly supported cohomology group $H_{\mathrm{c}}^{q}\left(U,\left.\mathcal{O}_{\lambda}\right|_{U}\right)$ defines, in a natural way, a nonzero submodule of $H_{\mathrm{c}}^{q}\left(S, \mathcal{O}_{\lambda} \mid s\right)$, which is irreducible (i.e., realizes the unique irreducible submodule of $H_{\mathrm{c}}^{q}\left(S, \mathcal{O}_{\lambda} \mid s\right)$ ) when an associated algebraic variety is nonsingular. By a tensoring argument, we can show that the result holds, more generally (for nonsingular associated variety), when the representation $H_{\mathrm{c}}^{q}\left(S, \mathcal{O}_{\lambda} \mid S\right)$ is what we call a classifying module.
\end{abstract}

\section{Introduction}

In this manuscript we show there is a natural generalization of the Borel-Weil theorem to the class of reductive Lie groups which serves to realize many, but not all, irreducible admissible representations.

Starting with Schmid's thesis [1989], there are general results realizing irreducible representations as sheaf cohomologies of finite-rank holomorphic vector bundles defined over open orbits in generalized complex flag spaces [Wong 1995; Bratten 1998]. However, relatively few irreducible representations can be realized this

Keywords: reductive Lie group, representation theory, flag manifold. 
way. The equivariant $\mathcal{D}$-module theory of Beilinson and Bernstein [1981] provides a powerful generalization to the Borel-Weil theorem and produces geometric realizations for any irreducible Harish-Chandra module. However, one would also like to find a natural realization of a corresponding group representation. In a general sense, the analytic localization defined by Hecht and Taylor [1990] does just that, by giving realizations for the minimal globalizations [Schmid 1985] of Harish-Chandra modules. Along these lines, the theory of analytic localization was used by Hecht and Taylor to realize minimal globalizations of the standard modules defined by the Beilinson-Bernstein theory. In many cases, standard modules are irreducible, but when they are not, it is not obvious how to proceed. One difficulty is that the geometric realization defined by Hecht and Taylor is obtained via an equivalence of derived categories so that the analytic localization of an irreducible representation can (and sometimes does) appear as a complex of sheaves that has nonzero homologies in various degrees. In spite of this difficulty, it turns out (somewhat surprisingly to us) that the theory of analytic localization can be used to realize many more irreducible representations than the example of irreducible standard modules. In the end, one sees that the key point hinges on whether a certain associated algebraic variety has singularities. When it does not, then the BeilinsonBernstein realization of the corresponding irreducible Harish-Chandra module has a simple geometric description and this fact controls the analytic localization. When the associated variety is singular, this simplicity breaks down, and it turns out that it is impossible to realize the irreducible representation as the sheaf cohomology of a finite-rank holomorphic vector bundle defined over an invariant open submanifold of a generalized flag manifold.

Rather than make a general statement about our main results in the introduction (our main results are Theorem 5.1 and Corollary 6.2), we would like to illustrate how the theory works in the context of a connected complex reductive group where the relationship to the Beilinson-Bernstein classification of irreducible admissible representations is more transparent. In particular, suppose $G_{0}$ is a connected complex reductive group with Lie algebra $\mathfrak{g}_{0}$ and let $K_{0} \subseteq G_{0}$ be a compact real form. Associated to $K_{0}$ is a corresponding Cartan involution $\theta: G_{0} \rightarrow G_{0}$ (in this case $\theta$ is the conjugation given by the real form). Let $X_{0}$ be the complex flag manifold of Borel subgroups of $G_{0}$, and let $X_{0}^{\mathrm{c}}$ be the conjugate complex manifold. Then the flag manifold $X$ of Borel subalgebras of the complexified Lie algebra $\mathfrak{g}$ of $\mathfrak{g}_{0}$ can be identified with the direct product

$$
X=X_{0} \times X_{0}^{\mathrm{c}}
$$

We need to consider two actions of $G_{0}$ on $X$. The diagonal action

$$
g \cdot(x, y)=(g x, g y)
$$


corresponds to the fact that $G_{0}$ is a real group with real Lie algebra $\mathfrak{g}_{0}$ and the action

$$
g \cdot(x, y)=(g x, \theta(g) y)
$$

corresponds to the fact that $G_{0}=K$ is the complexification of $K_{0}$. Choose a $\theta$-stable Cartan subgroup $H_{0} \subseteq G_{0}$ and a Borel subgroup $B_{0} \supseteq H_{0}$. Let $W\left(G_{0}\right)$ be the Weyl group of $H_{0}$ in $G_{0}$. Then we can identify the set of Borel subgroups of $G_{0}$ that contain $H_{0}$ with $W\left(G_{0}\right)$ (the identity in $W\left(G_{0}\right)$ corresponds to the Borel subgroup $\left.B_{0}\right)$. Let $B_{0}^{\text {op }}$ be the Borel subgroup opposite to $B_{0}$ with respect to $H_{0}$ (this subgroup corresponds to the longest element in $W\left(G_{0}\right)$ ). Then each $G_{0}$-orbit and each $K$-orbit on $X$ contains exactly one point of the form

$$
\left(w \cdot B_{0}, B_{0}^{\mathrm{op}}\right) \in X_{0} \times X_{0}^{\mathrm{c}}
$$

for $w \in W\left(G_{0}\right)$. Thus, the orbits for both actions are simultaneously parametrized by $W\left(G_{0}\right)$. Observe that the open orbit for the $G_{0}$-action and the closed orbit for the $K$-action correspond to the identity in $W\left(G_{0}\right)$ We introduce the length function, $l(w)$, on $W\left(G_{0}\right)$. In particular, each element $w \in W\left(G_{0}\right)$ can be expressed as a product of simple reflections and the corresponding length, $l(w)$, is defined to be the number of simple reflections that appear in a minimal expression (i.e., a reduced word) for $w$. Observe that if $Q_{w}$ is the $K$-orbit corresponding to $w \in W\left(G_{0}\right)$ then the complex dimension of $Q_{w}$ is given by

$$
\operatorname{dim}_{\mathbb{C}}\left(Q_{w}\right)=\operatorname{dim}_{\mathbb{C}}\left(X_{0}\right)+l(w) .
$$

For simplicity we will consider the sheaf of holomorphic functions $\mathcal{O}_{X}$ on $X$ (more generally one could consider the sheaf of sections $\mathcal{O}_{\lambda}$ of a $G_{0}$-equivariant holomorphic line bundle on $X$ whose parameter $\lambda$ in the usual twisted $\mathcal{D}$-module context is antidominant and regular). The Beilinson-Bernstein classification gives a one-to-one correspondence between the equivalence classes of irreducible admissible representations for $G_{0}$ that have the same infinitesimal character as the trivial representation and the $G_{0}$-orbits on $X$ given in the following way. For $w \in W\left(G_{0}\right)$, let $S_{w}$ be the corresponding $G_{0}$-orbit and define

$$
q=\operatorname{dim}_{\mathbb{C}}\left(X_{0}\right)-l(w) .
$$

Thus, $q$ is the (complex) codimension of the $K$-orbit $Q_{w}$ in $X$. Using their theory of analytic localization, Hecht and Taylor have shown that the compactly supported sheaf cohomologies

$$
H_{\mathrm{c}}^{p}\left(S_{w},\left.\mathcal{O}_{X}\right|_{S_{w}}\right)
$$

of the restriction of $\mathcal{O}_{X}$ to $S_{w}$ vanish except when $p=q$, in which case the module $H_{\mathrm{c}}^{q}\left(S_{w}, \mathcal{O}_{X} \mid S_{w}\right)$ is the minimal globalization of a corresponding standard Beilinson-Bernstein module. It follows that $H_{\mathrm{c}}^{q}\left(S_{w},\left.\mathcal{O}_{X}\right|_{S_{w}}\right)$ has a unique 
irreducible submodule $J_{w} \subseteq H_{\mathrm{c}}^{q}\left(S_{w},\left.\mathcal{O}_{X}\right|_{S_{w}}\right)$. These representations $J_{w}$ for $w \in W\left(G_{0}\right)$ are exactly the irreducible admissible representations for $G_{0}$ that have the same infinitesimal character as the trivial representation.

In this manuscript we want to realize the representations $J_{w}$. Along those lines we introduce the Bruhat order in $W\left(G_{0}\right)$ : if $w, u \in W\left(G_{0}\right)$ then we write $u \preceq w$ if $u$ is an ordered subword that occurs in a reduced expression for $w$ in terms of products of simple reflections. Given $w \in W\left(G_{0}\right)$, it is well known that the Bruhat interval $\Upsilon(w)=\left\{u \in W\left(G_{0}\right): u \leq w\right\}$ characterizes the Zariski closure of the $K$-orbit $Q_{w}$ in the following way:

$$
\overline{Q_{w}}=\bigcup_{u \in \Upsilon(w A)} Q_{u} .
$$

We call $\overline{Q_{w}}$ the algebraic variety associated to the $G_{0}$-orbit $S_{w}$. Define

$$
U_{w}=\bigcup_{u \in \Upsilon(w)} S_{u}
$$

Then, $U_{w}$ is the smallest $G_{0}$-invariant open submanifold of $X$ that contains $S_{w}$ and it is not hard to show that $S_{w}$ is the unique $G_{0}$-orbit that is closed in $U_{w}$. Put $U=U_{w}-S_{w}$. Letting $\left(\left.\mathcal{O}_{X}\right|_{U}\right)^{X}$, etc., denote the extension by zero of the restriction of $\mathcal{O}_{X}$ to $U$, we obtain the following short exact sequence of sheaves on $X:$

$$
0 \rightarrow\left(\left.\mathcal{O}_{X}\right|_{U}\right)^{X} \rightarrow\left(\left.\mathcal{O}_{X}\right|_{U_{w}}\right)^{X} \rightarrow\left(\left.\mathcal{O}_{X}\right|_{S_{w}}\right)^{X} \rightarrow 0 .
$$

Using an argument like [Bratten 2008, Lemma 3.3], it is not hard to show that

$$
H_{\mathrm{c}}^{p}\left(U,\left.\mathcal{O}_{X}\right|_{U}\right)=0 \quad \text { if } p<q+1
$$

and that

$$
H_{\mathrm{c}}^{q}\left(U_{w},\left.\mathcal{O}_{X}\right|_{U_{w}}\right)
$$

is a nonzero minimal globalization. Thus, the long exact sequence in sheaf cohomology determines an inclusion

$$
H_{\mathrm{c}}^{q}\left(U_{w},\left.\mathcal{O}_{X}\right|_{U_{w}}\right) \hookrightarrow H_{\mathrm{c}}^{q}\left(S_{w},\left.\mathcal{O}_{X}\right|_{S_{w}}\right) .
$$

We note that when $U_{w}$ is the preimage of an open $G_{0}$-orbit on a generalized flag space $Y$ then there is a natural identification of the representation $H_{\mathrm{c}}^{q}\left(U_{w},\left.\mathcal{O}_{X}\right|_{U_{w}}\right)$ with the $q$-th compactly supported cohomology of the holomorphic functions on the given open orbit in $Y$. (This is one of the key points in [op. cit.].) When this happens, it is known that the representation $H_{\mathrm{c}}^{q}\left(U_{w},\left.\mathcal{O}_{X}\right|_{U_{w}}\right)$ is irreducible. We say that $U_{w}$ is parabolic when $U_{w}$ is the preimage of an open $G_{0}$-orbit on a generalized flag space $Y$. Our main result in this study shows that (more generally) the submodule $H_{\mathrm{c}}^{q}\left(U_{w},\left.\mathcal{O}_{X}\right|_{U_{w}}\right)$ is irreducible when the associated algebraic variety $\overline{Q_{w}}$ is smooth. 
Thus we can realize $J_{w}$ as $H_{\mathrm{c}}^{q}\left(U_{w},\left.\mathcal{O}_{X}\right|_{U_{w}}\right)$ when (and, in fact, only when) this happens.

For example, if $G_{0}$ is the complex general linear group $\operatorname{GL}(3, \mathbb{C})$, then 4 out of the $6 G_{0}$-orbits on $X$ are parabolic but the algebraic varieties associated to all 6 orbits are smooth so we can realize all irreducible representations with the given infinitesimal character in this case. If $G_{0}=\mathrm{GL}(4, \mathbb{C})$, then only 8 of the $24 G_{0}$-orbits are parabolic, but 22 out of 24 orbits have smooth associated varieties so we can realize all but two of the irreducible representations with the given infinitesimal character (and so on). We will also see (for some examples) that when the algebraic variety $\overline{Q_{w}}$ is singular the representation $H_{\mathrm{c}}^{q}\left(U_{w},\left.\mathcal{O}_{X}\right|_{U_{w}}\right)$ is reducible, and it is actually impossible to realize the irreducible representation $J_{w}$ as the compactly supported sheaf cohomology of an equivariant (finite-rank) holomorphic vector bundle defined on a $G_{0}$-invariant open submanifold in a generalized flag space.

Our manuscript is organized as follows. In Section 2, we will present the main results we need about orbits and invariant subspaces in $X$. In Section 3, we will introduce the equivariant homogeneous line bundles and prove the basic embedding theorem. In Section 4, we introduce the algebraic localization theory and give a geometric description to the irreducible Harish-Chandra module in the BeilinsonBernstein classification, assuming the corresponding algebraic variety is smooth. In Section 5, we introduce the analytic localization and use the comparison theorem to prove our main result. Then, in Section 6, we use a tensoring argument to extend our result to antidominant parameters and also consider how our construction relates to the classical parabolic induction (in the case of a complex reductive group) so we can consider some examples. We conclude our manuscript with a brief consideration of how Serre duality applies. We would like to mention that the idea of our proof involves a mix of ideas from the two articles [Bratten 2008; 1997]. Although our argument requires a heavy use of the $D$-module theory and some familiarity with derived categories, we would hope it looks natural to anyone familiar with these two previous articles.

\section{2. $G_{0}$-orbits and $K$-orbits}

Throughout this manuscript $G_{0}$ will denote a real reductive Lie group of HarishChandra class with Lie algebra $\mathfrak{g}_{0}$ and complexified Lie algebra $\mathfrak{g}$. Abusing notation a bit, we let $G$ denote the complex adjoint group of $\mathfrak{g}$. (Note that $G$ has Lie algebra $[\mathfrak{g}, \mathfrak{g}]$.) There is a natural morphism of Lie groups

$$
G_{0} \rightarrow G
$$

We also fix a maximal compact subgroup $K_{0} \subseteq G_{0}$ and let $K$ be the complexification of $K_{0}$. Associated to the maximal compact subgroup, there is an involutive 
automorphism

$$
\theta: G_{0} \rightarrow G_{0}
$$

whose fixed point set is $K_{0}$. The involution $\theta$ (as well as the complexification $\theta: \mathfrak{g} \rightarrow \mathfrak{g}$ of its derivative) is called the Cartan involution.

A Borel subalgebra of $\mathfrak{g}$ is a maximal solvable subalgebra. $G$ acts transitively on the set of Borel subalgebras of $\mathfrak{g}$ and the resulting homogeneous $G$-space $X$ is a complex projective variety called the full flag space of $G_{0}$. Since $G_{0}$ has finitely many orbits on $X$ [Wolf 1969], the $G_{0}$-orbits are locally closed submanifolds.

A basic geometric property of flag manifolds that is fundamental to our study is the existence of a one-to-one correspondence between $G_{0}$-orbits and $K$-orbits referred to as Matsuki duality. For $x \in X$ we let $\mathfrak{b}_{x}$ denote the corresponding Borel subalgebra of $\mathfrak{g}$. Then, the nilradical $\mathfrak{n}_{x}$ of $\mathfrak{b}_{x}$ is given by $\mathfrak{n}_{x}=\left[\mathfrak{b}_{x}, \mathfrak{b}_{x}\right]$. The point $x \in X$ (as well as the Borel subalgebra $\mathfrak{b}_{x}$ ) is called special if there is a Cartan subalgebra $\mathfrak{c}$ of $\mathfrak{b}_{x}$ such that

$$
\mathfrak{c}_{0}=\mathfrak{g}_{0} \cap \mathfrak{c} \text { is a real form of } \mathfrak{c} \text { and } \theta(\mathfrak{c})=\mathfrak{c} .
$$

Matsuki [1979] showed that both the special points in a $G_{0}$-orbit as well as the special points in a $K$-orbit form a (nonempty) $K_{0}$-orbit. A $G_{0}$-orbit $S$ and a $K$-orbit $Q$ are said to be Matsuki dual if $S \cap Q$ contains a special point. It follows that Matsuki duality defines a bijection between the set of $G_{0}$-orbits and the set of $K$-orbits. When $S$ is a $G_{0}$-orbit and $Q$ is a $K$-orbit then we will write $S \sim Q$ when $S$ and $Q$ are Matsuki dual.

Given a $K$-orbit $Q$, the associated algebraic variety is defined to be the Zariski closure $\bar{Q}$ of $Q$ (when $S$ is the $G_{0}$-orbit dual $Q$, we will also refer to $\bar{Q}$ as the algebraic variety associated to $S$ ). There is a partial order, called the closure order, defined on the set of $K$-orbits by

$$
Q_{1} \preceq Q_{2} \text { if } Q_{1} \subseteq \overline{Q_{2}} .
$$

While the associated algebraic variety is a closed $K$-invariant subvariety of $X$ associated to a $G_{0}$-orbit $S$, we will now define a corresponding $G_{0}$-invariant open submanifold of $X$. In particular, suppose $S_{0}$ is a $G_{0}$-orbit and let $Q_{0}$ be the Matsuki dual. We define an associated indexing set $\Upsilon\left(S_{0}\right)$ of $G_{0}$-orbits by

$$
S \in \Upsilon\left(S_{0}\right) \Longleftrightarrow \exists Q \text { such that } Q \sim S \text { and } Q \subseteq \overline{Q_{0}} \text {. }
$$

We define the corresponding $G_{0}$-invariant subspace $U$ by

$$
U=\bigcup_{S \in \Upsilon\left(S_{0}\right)} S .
$$


Proposition 2.1. With the previous notation, $U$ is the smallest $G_{0}$-invariant open submanifold that contains $S_{0}$, and $S_{0}$ is the unique closed $G_{0}$-orbit in $U$.

Proof. We consider the closure orders on the set of $K$-orbits and on the set of $G_{0}$-orbits. Matsuki [1988] showed that duality reverses the corresponding closure relations. It follows that

$$
S \in \Upsilon\left(S_{0}\right) \quad \Longleftrightarrow \quad S_{0} \subseteq \bar{S} \Longleftrightarrow S_{0} \preceq S .
$$

Thus, if $S \notin \Upsilon\left(S_{0}\right)$, then $S_{0} \cap \bar{S}=\varnothing$; therefore, $S_{0} \cap \overline{S_{1}}=\varnothing$ for each $G_{0}$-orbit $S_{1}$ contained in $\bar{S}$. Hence,

$$
U \cap \bar{S}=\varnothing .
$$

Since there are a finite number of orbits, the set

$$
C=\bigcup_{S \notin \Upsilon\left(S_{0}\right)} \bar{S}
$$

is closed and therefore

$$
U=X-C
$$

is open.

Now, suppose that $W$ is an open $G_{0}$-invariant submanifold that contains $S_{0}$. Suppose $S \subseteq U$. Then $S_{0} \subseteq \bar{S}$. Thus,

$$
\bar{S} \cap W \neq \varnothing .
$$

Hence, $\bar{S} \cap W$ is a nonempty open $G_{0}$-invariant subset of $\bar{S}$. Since $S$ is locally closed, it follows that $S$ is open and dense in $\bar{S}$. Hence, from the $G_{0}$-invariance,

$$
S \subseteq \bar{S} \cap W \quad \Longrightarrow \quad S \subseteq W
$$

so that $U \subseteq W$, which proves that $U$ is the smallest $G_{0}$-invariant open submanifold that contains $S_{0}$.

To prove the last claim, first observe that if $S$ is a $G_{0}$-orbit contained in $\overline{S_{0}}$ then $S \preceq S_{0}$ so that $S \subseteq U$ if and only if $S=S_{0}$. Thus,

$$
\overline{S_{0}} \cap U=S_{0}
$$

and $S_{0}$ is closed in $U$. On the other hand, if $S$ is a closed $G_{0}$-orbit in $U$, then

$$
S=\bar{S} \cap U .
$$

However, from the definition of $U$, for $S \subseteq U$, we have $S_{0} \subseteq \bar{S}$, hence

$$
S_{0} \subseteq \bar{S} \cap U \text {. }
$$

It follows that $S=\bar{S} \cap U$ if and only if $S=S_{0}$. 
Example 2.2. Suppose that $\mathfrak{p} \subseteq \mathfrak{g}$ is a parabolic subalgebra and let $Y$ be the corresponding $G$-homogeneous space of parabolic subalgebras of $\mathfrak{g}$ conjugate to $\mathfrak{p}$. For each $y \in Y$, let $\mathfrak{p}_{y}$ denote the corresponding parabolic subalgebra of $\mathfrak{g}$. For $x \in X$, there is a unique $y \in Y$ such that $\mathfrak{b}_{x} \subseteq \mathfrak{p}_{y}$. Thus there is a canonical $G$-equivariant projection

$$
\pi: X \rightarrow Y
$$

given by $\pi(x)=y$ if $\mathfrak{b}_{x} \subseteq \mathfrak{p}_{y}$. A point $y \in Y$ is called special if $\mathfrak{p}_{y}$ contains a special Borel subalgebra.

Suppose $W \subseteq Y$ is an open $G_{0}$-orbit and let $y \in W$ be a special point. Let $O$ be the $K$-orbit of $y$. Then, $O$ is closed in $Y$ (in fact $O \subseteq W$ ), and Matsuki [1982] showed that the $G_{0}$-orbits in $U=\pi^{-1}(W)$ are Matsuki dual to the $K$-orbits in $\pi^{-1}(O)$. Also, there is a unique $G_{0}$-orbit $S_{0}$ that is closed in $U$ and its Matsuki dual $Q_{0}$ is the unique open orbit in the closed algebraic variety $\pi^{-1}(O)$. Therefore, $\overline{Q_{0}}=\pi^{-1}(O)$ and it follows that a $G_{0}$-orbit $S$ is contained in $U$ if and only if its Matsuki dual $Q$ is contained in $\overline{Q_{0}}$; thus, $U$ is the smallest $G_{0}$-invariant open submanifold that contains $S_{0}$. Observe that, in this case, the associated algebraic variety $\overline{Q_{0}}=\pi^{-1}(O)$ is smooth since the fibers of $\pi$ are smooth and since $\pi$ defines a locally trivial algebraic fibration of $\pi^{-1}(O)$ over $O$.

\section{Equivariant line bundles}

In this section, we introduce the equivariant line bundles on the full flag space $X$, as well as the corresponding standard modules associated to a $G_{0}$-orbit $S \subseteq X$. We begin this section by introducing the abstract Cartan dual, which is the parameter set for the twisted sheaves of differential operators (TDOs) on $X$. Recall that $G$ is the complex adjoint group of $\mathfrak{g}$. For $x \in X$, let $\mathfrak{n}_{x}$ denote the nilradical of the corresponding Borel subalgebra $\mathfrak{b}_{x}$ and put

$$
\mathfrak{h}_{x}=\mathfrak{b}_{x} / \mathfrak{n}_{x}
$$

Since the stabilizer of $x$ in $G$ (i.e., the corresponding Borel subgroup in $G$ ) acts trivially on $\mathfrak{h}_{x}$ it also acts trivially on the complex dual $\mathfrak{h}_{x}^{*}$. It follows that the corresponding $G$-homogeneous holomorphic vector bundle on $X$ is trivial and that the associated space of global sections $\mathfrak{h}^{*}$ is naturally isomorphic to $\mathfrak{h}_{x}^{*}$ via the evaluation at $x$. The vector space $\mathfrak{h}^{*}$ is called the abstract Cartan dual for $\mathfrak{g}$. If $\mathfrak{c}$ is a Cartan subalgebra of $\mathfrak{b}_{x}$, then by coupling the natural projection of $\mathfrak{c}$ onto $\mathfrak{h}_{x}$ with the evaluation at $x$, we obtain an isomorphism of $\mathfrak{c}^{*}$ with $\mathfrak{h}^{*}$ called the specialization of $\mathfrak{h}^{*}$ to $\mathfrak{c}^{*}$ at $x$. Using the specializations, we can identify an abstract set of roots

$$
\Sigma \subseteq \mathfrak{h}^{*}
$$


and an abstract set of positive roots

$$
\Sigma^{+} \subseteq \Sigma
$$

where $\Sigma$ corresponds to the set of roots of $\mathfrak{c}$ in $\mathfrak{g}$ and $\Sigma^{+}$corresponds to the roots of $\mathfrak{c}$ in $\mathfrak{b}_{x}$, via the specialization at $x$. Given $\alpha \in \Sigma$ and $\mu \in \mathfrak{h}^{*}$, we can also define the complex number

$$
\stackrel{\vee}{\alpha}(\mu),
$$

the value of $\mu$ on the coroot of $\alpha$. The element $\mu \in \mathfrak{h}^{*}$ is called integral if

$$
\stackrel{\vee}{\alpha}(\mu) \in \mathbb{Z} \quad \text { for each } \alpha \in \Sigma \text {. }
$$

It just so happens that the half-sum of positive roots, denoted by $\rho$, is an integral element of $\mathfrak{h}^{*}$ that plays a key role in the TDO parametrization.

Let $\widetilde{G}$ denote the universal cover of $G$ and suppose $\mu \in \mathfrak{h}^{*}$ is integral. For a point $x \in X$, the Lie algebra of the corresponding Borel subgroup $\widetilde{B}_{x}$ in $\widetilde{G}$ (i.e., the stabilizer of $x$ in $\widetilde{G}$ ) is given by $\mathfrak{b}_{x} \cap[\mathfrak{g}, \mathfrak{g}]$. Thus, using the evaluation at $x$, the global section $\mu$ determines a one-dimensional representation

$$
\mathfrak{b}_{x} \cap[\mathfrak{g}, \mathfrak{g}] \longrightarrow \mathfrak{h}_{x} \stackrel{\mu_{x}}{\longrightarrow} \mathbb{C} .
$$

Since $\widetilde{G}$ is simply connected, it is known that there is a (unique) holomorphic character of $\widetilde{B}_{x}$ whose derivative is given, in this way, by $\mu_{x}$. Thus, corresponding to each integral $\mu \in \mathfrak{h}^{*}$ there is a corresponding $\widetilde{G}$-homogeneous holomorphic line bundle

$$
\mathbb{L}(\mu) \longrightarrow X \text {. }
$$

Let $\mathcal{O}(\mu)$ be the corresponding sheaf of holomorphic sections. In a natural way, $\widetilde{G}$ and thus $[\mathfrak{g}, \mathfrak{g}]$ act on $\mathcal{O}(\mu)$. Let $\mathfrak{z}$ be the center of $\mathfrak{g}$. Suppose $W \subseteq X$ is an open set, and let

$$
\sigma: W \rightarrow \mathbb{L}(\mu)
$$

be a local holomorphic section. Then, extend $\mathcal{O}(\mu)$ to a sheaf of $\mathfrak{g}$-modules by

$$
(\xi \cdot \sigma)(x)=\mu(\xi) \sigma(x) \text { for } \xi \in \mathfrak{z} \text { and } x \in W .
$$

We say that $\mathbb{L}(\mu)$ is a $G_{0}$-equivariant line bundle if there exists a $G_{0}$-action on $\mathbb{L}(\mu)$ (in the sense of differentiable $G_{0}$-actions on vector bundles over differentiable $G_{0}$-spaces) such that the induced morphisms $\mathbb{L}(\mu) \rightarrow \mathbb{L}(\mu)$, given by multiplication by group elements, are holomorphic and such that the derivative of the $G_{0}$-action on local sections coincides with the $\mathfrak{g}$-action.

Example 3.1. An important class of $G_{0}$-equivariant holomorphic line bundles corresponds to the family of (equivalence classes of) finite-dimensional irreducible representations of $G_{0}$ that are also irreducible for the corresponding $\mathfrak{g}$-action (i.e., 
irreducible finite-dimensional $\mathfrak{g}$-modules with a compatible $G_{0}$-action). In particular, let $V$ be a finite-dimensional $G_{0}$-module that is irreducible as a $\mathfrak{g}$-module. For each $x \in X$, let $G_{0}[x]$ denote the stabilizer of $x$ and consider the corresponding $\left(\mathfrak{b}_{x}, G_{0}[x]\right)$-module

$$
V / \mathfrak{n}_{x} V
$$

Choosing a Cartan subalgebra $\mathfrak{c} \subseteq \mathfrak{b}_{x}$ and using the specialization to $x$, the action of $\mathfrak{c}$ on $V / \mathfrak{n}_{x} V$ is given by an element $\mu \in \mathfrak{h}^{*}$ that corresponds to the lowest weight in $V$. Hence, if we define

$$
\lambda=\mu-\rho
$$

then $\alpha(\lambda)$ is a negative integer, for each positive root $\alpha \in \Sigma^{+}$. We can define the total space of a $G_{0}$-equivariant holomorphic line bundle $\mathbb{L}_{\lambda}$ by

$$
\mathbb{L}_{\lambda}=\bigcup_{x \in X} V / \mathfrak{n}_{x} V .
$$

Using the action of $\widetilde{G}$ one can define holomorphic transition functions. Then the Borel-Weil Theorem says that the representation $V$ is recovered as the global holomorphic sections of the bundle $\mathbb{L}_{\lambda}$.

In general,, when $\mathcal{O}(\mu)$ is the sheaf of holomorphic sections of $G_{0}$-equivariant line bundle we will use the shifted parameter $\lambda=\mu-\rho$ and write

$$
\mathcal{O}_{\lambda}=\mathcal{O}(\mu)
$$

for the sheaf of holomorphic sections. We say $\lambda$ is regular if

$$
\stackrel{\vee}{\alpha}(\lambda) \neq 0 \quad \text { for each } \operatorname{root} \alpha \in \Sigma \text {. }
$$

An element $\lambda$ is called singular when it is not regular. We say $\lambda$ is antidominant if

$$
\stackrel{\vee}{\alpha}(\lambda) \notin \mathbb{N} \text { for each positive root } \alpha \in \Sigma^{+} \text {. }
$$

Suppose that $\mathcal{O}_{\lambda}$ is the sheaf of holomorphic sections of a $G_{0}$-equivariant line bundle. Let $S$ be a $G_{0}$-orbit in $X$ and let $Q$ be the Matsuki dual to $S$. We define the vanishing number $q$ of $S$ to be the (complex) codimension of $Q$ in $X$. Suppose that $\lambda$ is antidominant and regular. One of the main results of the Hecht-Taylor analytic localization theory is that the compactly supported sheaf cohomology of the restriction $\left.\mathcal{O}_{\lambda}\right|_{S}$ of $\mathcal{O}_{\lambda}$ to $S$ vanishes, except in degree $q$, in which case

$$
H_{\mathrm{c}}^{q}\left(S, \mathcal{O}_{\lambda} \mid S\right)
$$

is the minimal globalization of a corresponding standard Beilinson-Bernstein module. (We will describe this module in the following section.) In particular, 
$H_{\mathrm{c}}^{q}\left(S,\left.\mathcal{O}_{\lambda}\right|_{S}\right)$ has a unique irreducible submodule. In general, (for any parameter $\lambda)$, the sheaf cohomology groups

$$
H_{\mathrm{c}}^{p}\left(S, \mathcal{O}_{\lambda} \mid S\right)
$$

vanish [Bratten 1997] for $p<q$, and in the nonzero cases these cohomology groups are minimal globalizations of the sheaf cohomology groups of an associated standard Harish-Chandra sheaf.

In general, terms, we now consider a simple geometric procedure which can be used in the context of the Hecht-Taylor realization of minimal globalizations, to study representations. We first remark that the sheaves $\mathcal{O}_{\lambda}$ are examples of what is referred to in the Hecht-Taylor development as DNF (stands for dual nuclear Fréchet) sheaves of analytic $G_{0}$-modules (we will not need the formalism of DNF sheaves of analytic $G_{0}$-modules in our study however we would simply like to mention the general criteria used to establish the following results). We want to remark that, in the case of the global sheaf cohomology on $X$, the sheaf cohomology groups

$$
H^{p}\left(X, \mathcal{O}_{\lambda}\right)
$$

are finite-dimensional and were originally studied in [Bott 1957]. Now suppose $L \subseteq X$ is a locally closed $G_{0}$-invariant subspace and let

$$
\left(\left.\mathcal{O}_{\lambda}\right|_{L}\right)^{X}
$$

denote the extension by zero to $X$ of the restriction of $\mathcal{O}_{\lambda}$ to $L$. Then there is a natural isomorphism of functors

$$
H^{p}\left(X,\left(\left.\mathcal{O}_{\lambda}\right|_{L}\right)^{X}\right) \cong H_{\mathrm{c}}^{p}\left(L,\left.\mathcal{O}_{\lambda}\right|_{L}\right)
$$

and it follows from the results of Hecht and Taylor (at least for $\lambda$ regular — in the singular case one can prove this by a tensoring argument as in [Bratten 1997]) that the sheaf cohomology groups

$$
H_{\mathrm{c}}^{p}\left(L,\left.\mathcal{O}_{\lambda}\right|_{L}\right)
$$

are minimal globalizations of Harish-Chandra modules. Let $W \subseteq L$ be an open, $G_{0}$-invariant subspace and let $C=L-W$. Then we have the following short exact sequence of DNF sheaves of analytic $G_{0}$-modules:

$$
0 \rightarrow\left(\left.\mathcal{O}_{\lambda}\right|_{W}\right)^{X} \rightarrow\left(\left.\mathcal{O}_{\lambda}\right|_{L}\right)^{X} \rightarrow\left(\left.\mathcal{O}_{\lambda}\right|_{C}\right)^{X} \rightarrow 0 .
$$

Therefore, the corresponding long exact sequence in cohomology

$\cdots \rightarrow H_{\mathrm{c}}^{p}\left(W,\left.\mathcal{O}_{\lambda}\right|_{W}\right) \rightarrow H_{\mathrm{c}}^{p}\left(L,\left.\mathcal{O}_{\lambda}\right|_{L}\right) \rightarrow H_{\mathrm{c}}^{p}\left(C,\left.\mathcal{O}_{\lambda}\right|_{C}\right) \rightarrow H_{\mathrm{c}}^{p+1}\left(W,\left.\mathcal{O}_{\lambda}\right|_{W}\right) \rightarrow \cdots$

is a sequence of minimal globalizations with continuous $G_{0}$-morphisms. 
Return to the case where $S$ is a $G_{0}$-orbit and let $U$ be the smallest $G_{0}$-invariant open set that contains $S$. Then the compactly supported sheaf cohomology groups

$$
H_{\mathrm{c}}^{p}\left(U,\left.\mathcal{O}_{\lambda}\right|_{U}\right)
$$

are minimal globalizations of Harish-Chandra modules. Recall that $q$ is the vanishing number of $S$. We will now show that there is a natural embedding of $H_{\mathrm{c}}^{q}\left(U,\left.\mathcal{O}_{\lambda}\right|_{U}\right)$ in $H_{\mathrm{c}}^{q}\left(S,\left.\mathcal{O}_{\lambda}\right|_{S}\right)$. Let $W=U-S$. Then $W$ is open and we have the following short exact sequence of sheaves on $X$ :

$$
0 \rightarrow\left(\left.\mathcal{O}_{\lambda}\right|_{W}\right)^{X} \rightarrow\left(\left.\mathcal{O}_{\lambda}\right|_{U}\right)^{X} \rightarrow\left(\left.\mathcal{O}_{\lambda}\right|_{S}\right)^{X} \rightarrow 0 .
$$

This sequence will induce a sequence of continuous morphisms of minimal globalizations when we apply the long exact sequence of sheaf cohomology. To prove we have an inclusion in grade $q$ we use the vanishing on $S$, and the following lemma.

Lemma 3.2. Maintain the previously defined notations. Then,

$$
H_{\mathrm{c}}^{p}\left(W,\left.\mathcal{O}_{\lambda}\right|_{W}\right)=0 \text { for } p \leq q .
$$

Proof. First observe that since $Q$ is open in $\bar{Q}$ then for each $Q_{0} \subseteq \bar{Q}$ such that $Q_{0} \neq Q$ then the codimension of $Q_{0}$ is strictly bigger than the codimension of $Q$, so that the vanishing numbers for $G_{0}$-orbits in $W$ are at least $q+1$. Suppose $O \subseteq W$ is a $G_{0}$-invariant open subset. We define the length of $O$ to be the number of $G_{0}$-orbits contained in $O$. We show that the announced vanishing result holds for every $G_{0}$-invariant open subset of $W$ by an induction on length. When $O$ has length one, it is an open $G_{0}$-orbit so the result holds since it has vanishing number at least $q+1$. In general, let $S_{0} \subseteq O$ be a $G_{0}$-orbit of minimal dimension. Since $S_{0}$ is open and dense in its closure it follows that any $G_{0}$-orbit in the closure different from $S_{0}$ has strictly smaller dimension. Thus,

$$
\overline{S_{0}} \cap O=S_{0}
$$

and $O_{0}=O-S_{0}$ is an open $G_{0}$-invariant of shorter length. Thus, the result follows by induction, using the long exact sequence in cohomology applied to the short exact sequence of sheaves:

$$
0 \rightarrow\left(\mathcal{O}_{\lambda} \mid o_{0}\right)^{X} \rightarrow\left(\mathcal{O}_{\lambda} \mid o\right)^{X} \rightarrow\left(\left.\mathcal{O}_{\lambda}\right|_{S_{0}}\right)^{X} \rightarrow 0 .
$$

Corollary 3.3. Let $\mathcal{O}_{\lambda}$ be the sheaf of sections of a $G_{0}$-equivariant holomorphic line bundle over $X$. Suppose $S \subseteq X$ is $G_{0}$-orbit with associated vanishing number $q$ and let $U$ be the smallest $G_{0}$-invariant open submanifold that contains $S$. Then there is a natural inclusion of analytic $G_{0}$-modules

$$
H_{\mathrm{c}}^{q}\left(U,\left.\mathcal{O}_{\lambda}\right|_{U}\right) \rightarrow H_{\mathrm{c}}^{q}\left(S,\left.\mathcal{O}_{\lambda}\right|_{S}\right)
$$




\section{Localization and standard Beilinson-Bernstein modules}

We begin this section by introducing the sheaves of twisted differential operators and reviewing some of the necessary theory. For a basic reference on the algebraic side of the localization theory, we note that [Miličić 1993] provides a nice overview to the geometric realization of Harish-Chandra modules given by the Beilinson-Bernstein theory.

Let $U(\mathfrak{g})$ be the enveloping algebra of $\mathfrak{g}$ and let $Z(\mathfrak{g})$ be the center of $U(\mathfrak{g})$. An infinitesimal character $\Theta$ is a morphism of algebras (with identity)

$$
\Theta: Z(\mathfrak{g}) \rightarrow \mathbb{C} .
$$

We let $U_{\Theta}$ be the quotient of $U(\mathfrak{g})$ by the two-sided generated from the kernel of $\Theta$ in $Z(\mathfrak{g})$. Observe that $U_{\Theta}$ is the algebra that acts naturally on a $\mathfrak{g}$-module with infinitesimal character $\Theta$.

Let $W$ be the Weyl group of $\mathfrak{h}^{*}$. By Harish-Chandra's classical result, $Z(\mathfrak{g})$ is isomorphic to the Weyl group invariants in the enveloping algebra of a Cartan subalgebra of $\mathfrak{g}$. It follows that the infinitesimal characters are naturally parametrized by the $W$-orbits in $\mathfrak{h}^{*}$. For $\lambda \in \mathfrak{h}^{*}$, we write

$$
\Theta=W \lambda \quad \text { and } \quad \lambda \in \Theta
$$

when the $W$ orbit of $\lambda$ parametrizes the infinitesimal character $\Theta$. It is known that if $\lambda \in \mathfrak{h}^{*}$ is integral (or regular) then $w \lambda$ is integral (or regular) for every $w \in W$. In this case we also say that the corresponding infinitesimal character is integral (or regular). When an infinitesimal character $\Theta$ is integral and regular then there exists a unique $\lambda \in \Theta$ that is antidominant. Notice that the infinitesimal character of an irreducible admissible representation is an important invariant and that the Beilinson-Bernstein realization of irreducible Harish-Chandra modules with infinitesimal character $\Theta$ depends, to some extent, on the choice of $\lambda \in \Theta$.

At this point we need to distinguish between the algebraic and analytic structures on $X$. Therefore, we consider the full flag space $X$ as both an algebraic variety (with the Zariski topology) and as a complex manifold (with the analytic topology) according to the context. Since the line bundles, defined for integral $\lambda \in \mathfrak{h}^{*}$ in the previous section, have a compatible algebraic structure, we can consider the corresponding sheaf of algebraic sections $\mathcal{O}_{\lambda}^{\text {alg }}$ defined on the algebraic variety $X$. Associated to the sheaf $\mathcal{O}_{\lambda}^{\text {alg }}$ is a corresponding twisted sheaf of differential operators (TDO) $\mathcal{D}_{\lambda}^{\mathrm{alg}}$.

We can also consider the corresponding TDO $\mathcal{D}_{\lambda}$, with holomorphic coefficients and defined on complex variety $X$. In a natural way, $\mathcal{O}_{\lambda}$ is a sheaf of modules for $\mathcal{D}_{\lambda}$. When $\mathcal{O}_{\lambda}$ is the sheaf of holomorphic sections of a $G_{0}$-equivariant line bundle, $G_{0}$ acts on $\mathcal{D}_{\lambda}$ while $K$ acts compatibly (extending the $K_{0}$-action) on $\mathcal{O}_{\lambda}^{\text {alg }}$ and 
$\mathcal{D}_{\lambda}^{\text {alg }}$. We want to emphasize that we are using the shifted parametrization from the previous section. In particular, $\mathcal{O}_{-}$is the sheaf of holomorphic functions on $X$ and $\mathcal{D}_{-} \rho$ is the sheaf of holomorphic differential operators.

Suppose that $\lambda \in \Theta$. Beilinson and Bernstein showed that

$$
\Gamma\left(X, \mathcal{D}_{\lambda}^{\mathrm{alg}}\right) \cong U_{\Theta} \quad \text { and } \quad H^{p}\left(X, \mathcal{D}_{\lambda}^{\mathrm{alg}}\right)=0 \text { for } p>0 .
$$

Thus, the sheaf cohomology groups of a sheaf of $\mathcal{D}_{\lambda}^{\text {alg }}$-modules are $\mathfrak{g}$-modules with infinitesimal character $\Theta$. When $\mathcal{F}$ is a sheaf of quasicoherent $\mathcal{D}_{\lambda}^{\mathrm{alg}}$-modules and $\lambda$ is antidominant, Beilinson and Bernstein showed that

$$
H^{p}(X, \mathcal{F})=0 \text { for } p>0 .
$$

In particular, when $\lambda$ is antidominant, the functor of global sections is exact on the category of quasicoherent $\mathcal{D}_{\lambda}^{\text {alg }}$-modules. The localization functor

$$
\Delta_{\lambda}(M)=\mathcal{D}_{\lambda}^{\mathrm{alg}} \otimes_{U_{\Theta}} M
$$

is defined on the category of $U_{\Theta}$-modules and is the left adjoint to the global sections functor on the category of quasicoherent $\mathcal{D}_{\lambda}^{\mathrm{alg}}$-modules. When $\lambda$ is antidominant and regular, Beilinson and Bernstein showed that the localization functor and the global sections functor are mutual inverses and determine an equivalence of categories.

Suppose $S \subseteq X$ is a $G_{0}$-orbit with associated vanishing number $q$. Let $\mathcal{O}_{\lambda}$ be the sheaf of holomorphic sections of a $G_{0}$-equivariant line bundle. We now consider the geometric construction of the underlying Harish-Chandra module of the minimal globalization

$$
H_{\mathrm{c}}^{q}\left(S,\left.\mathcal{O}_{\lambda}\right|_{S}\right) .
$$

Let $Q$ denote the $K$-orbit Matsuki dual to $S$ (equip $Q$ with the Zariski topology) and consider the $K$-equivariant sheaf $\mathcal{O}_{\lambda}^{\text {alg }}$. Let $i: Q \hookrightarrow X$ be the inclusion and let

$$
i^{*}\left(\mathcal{O}_{\lambda}^{\mathrm{alg}}\right)=i^{-1}\left(\mathcal{O}_{\lambda}^{\mathrm{alg}}\right) \otimes_{i^{-1}}\left(\mathcal{O}_{X}^{\mathrm{alg}}\right) \mathcal{O}_{Q}^{\mathrm{alg}}
$$

be the inverse image of $\mathcal{O}_{\lambda}^{\text {alg }}$ with respect to the structure sheaves of the algebraic varieties $Q$ and $X$. Therefore, $i^{*}\left(\mathcal{O}_{\lambda}^{\mathrm{alg}}\right)$ is the sheaf of sections of a corresponding $K$-homogeneous algebraic line bundle defined on $Q$. Let $\mathcal{D}_{Q, \lambda}^{\text {alg }}$ be the sheaf of differential operators for the locally free sheaf $i^{*}\left(\mathcal{O}_{\lambda}^{\text {alg }}\right)$. Then, there is a corresponding direct image functor $i_{+}$in the category of sheaves of (twisted) $\mathcal{D}$-modules. In this case, since the morphism $i$ is an affine inclusion of smooth varieties, the direct image is an exact functor [Hecht et al. 1987]. The sheaf

$$
\mathcal{I}(Q, \lambda)=i_{+} i^{*}\left(\mathcal{O}_{\lambda}^{\text {alg }}\right)
$$

is a $K$-equivariant sheaf of $\mathcal{D}_{\lambda}^{\text {alg }}$-modules called the corresponding standard HarishChandra sheaf on $X$, which contains a unique (coherent and $K$-invariant) irreducible 
subsheaf

$$
\mathcal{J}(Q, \lambda) \subseteq \mathcal{I}(Q, \lambda)
$$

of $\mathcal{D}_{\lambda}^{\text {alg }}$-modules. We note that the notation being used is a bit ambiguous since the structure of these objects also depends on the $K_{0}$-action and not just the orbit $Q$ and the integral parameter $\lambda$. However, in the current context it should be clear how one construction leads to the other and we feel our approach avoids an overly complicated notation. The Harish-Chandra module

$$
I(Q, \lambda)=\Gamma\left(X, i_{+} i^{*}\left(\mathcal{O}_{\lambda}^{\mathrm{alg}}\right)\right)
$$

is called the corresponding standard Beilinson-Bernstein module. In general, one knows [Bratten 1997] that $I(Q, \lambda)$ is the underlying Harish-Chandra module of $H_{\mathrm{c}}^{q}\left(S,\left.\mathcal{O}_{\lambda}\right|_{S}\right)$. Observe that when $\lambda$ is antidominant and regular, by the equivalence of categories, it follows that the Harish-Chandra module

$$
J(Q, \lambda)=\Gamma(X, \mathcal{J}(Q, \lambda)) \subseteq I(Q, \lambda)
$$

is the unique irreducible submodule of the corresponding standard BeilinsonBernstein module. We call $I(Q, \lambda)$ a classifying module if $\lambda$ is antidominant and $J(Q, \lambda) \neq 0$. As the name suggests, the classifying modules are used in Beilinson-Bernstein classification of irreducible admissible representations. This works perfectly when $G_{0}$ is a connected, complex reductive group, however, in general, one must enlarge the class of standard Harish-Chandra sheaves to include all irreducible representations with the given integral infinitesimal character.

Suppose $\lambda$ is antidominant and regular and let $J(Q, \lambda)_{\min }$ denote the corresponding minimal globalization.

Proposition 4.1. Maintain the above notations (in particular, we assume that $\lambda$ $i$ is antidominant and regular). Let $U$ be the smallest $G_{0}$-invariant open set that contains $S$. Then, there exists a natural inclusion

$$
J(Q, \lambda)_{\min } \hookrightarrow H_{\mathrm{c}}^{q}\left(U,\left.\mathcal{O}_{\lambda}\right|_{U}\right) .
$$

Proof. Since $I(Q, \lambda)$ is the underlying Harish-Chandra module of $H_{\mathrm{c}}^{q}\left(S, \mathcal{O}_{\lambda} \mid S\right)$, it follows that $J(Q, \lambda)_{\min }$ is the unique irreducible submodule in $H_{\mathrm{c}}^{q}\left(S, \mathcal{O}_{\lambda} \mid S\right)$. Therefore, to establish the result it suffices to show that

$$
H_{\mathrm{c}}^{q}\left(U,\left.\mathcal{O}_{\lambda}\right|_{U}\right) \neq 0 .
$$

We follow the setup used in Lemma 3.2 and argue by contradiction. Suppose that $H_{\mathrm{c}}^{q}\left(U,\left.\mathcal{O}_{\lambda}\right|_{U}\right)=0$ and let $W=U-S$. Using the long exact sequence in cohomology, we obtain an inclusion

$$
J(Q, \lambda)_{\min } \hookrightarrow H_{\mathrm{c}}^{q}\left(S,\left.\mathcal{O}_{\lambda}\right|_{S}\right) \hookrightarrow H_{\mathrm{c}}^{q+1}\left(W,\left.\mathcal{O}_{\lambda}\right|_{W}\right)
$$


Now, suppose that $S_{1} \subseteq W$ is a $G_{0}$-orbit of minimal dimension and define $W_{1}=$ $W-S$. Then $S_{1}$ is closed in $W_{1}$, so using the corresponding long exact sequence in cohomology, we obtain the sequence

$$
0 \rightarrow H_{\mathrm{c}}^{q+1}\left(W_{1},\left.\mathcal{O}_{\lambda}\right|_{W_{1}}\right) \rightarrow H_{\mathrm{c}}^{q+1}\left(W,\left.\mathcal{O}_{\lambda}\right|_{W}\right) \rightarrow H_{\mathrm{c}}^{q+1}\left(S_{1},\left.\mathcal{O}_{\lambda}\right|_{S_{1}}\right) \rightarrow \cdots .
$$

Since $J(Q, \lambda)_{\min }$, is irreducible either

$$
J(Q, \lambda)_{\min } \hookrightarrow H_{\mathrm{c}}^{q+1}\left(W_{1},\left.\mathcal{O}_{\lambda}\right|_{W_{1}}\right) \quad \text { or } \quad J(Q, \lambda)_{\min } \hookrightarrow H_{\mathrm{c}}^{q+1}\left(S_{1},\left.\mathcal{O}_{\lambda}\right|_{S_{1}}\right) .
$$

However, if $H_{\mathrm{c}}^{q+1}\left(S_{1},\left.\mathcal{O}_{\lambda}\right|_{S_{1}}\right) \neq 0$, then this representation is the minimal globalization of the classifying module $I\left(Q_{1}, \lambda\right)$ where $Q_{1}$ is the $K$-orbit Matsuki dual to $S_{1}$. Thus, $J\left(Q_{1}, \lambda\right)_{\min }$ is the unique irreducible submodule of $H_{\mathrm{c}}^{q+1}\left(S_{1}, \mathcal{O}_{\lambda} \mid S_{1}\right)$. Since $Q_{1} \neq Q$, it follows that $J(Q, \lambda)$ is not isomorphic to $J\left(Q_{1}, \lambda\right)$. Therefore,

$$
J(Q, \lambda)_{\min } \hookrightarrow H_{\mathrm{c}}^{q+1}\left(W_{1},\left.\mathcal{O}_{\lambda}\right|_{W_{1}}\right) .
$$

Proceeding in this fashion, we would obtain that

$$
J(Q, \lambda)_{\min } \hookrightarrow H_{\mathrm{c}}^{q+1}\left(O, \mathcal{O}_{\lambda} \mid o\right),
$$

where $O$ is an open $G_{0}$-orbit. However, this is impossible since $H_{\mathrm{c}}^{q+1}\left(O, \mathcal{O}_{\lambda} \mid O\right)$ is either zero or an irreducible minimal globalization that is not isomorphic to $J(Q, \lambda)_{\min }$.

The proof of our main result now consists of two steps. The first part is to characterize the irreducible Harish-Chandra sheaf $\mathcal{J}(Q, \lambda)$ when the associated variety $\bar{Q}$ is smooth. Once we have that in hand, it turns out to be fairly straightforward to calculate the analytic localization of $J(Q, \lambda)_{\min }$ on $G_{0}$-orbits. To finish the proof we show that the inclusion

$$
J(Q, \lambda)_{\min } \hookrightarrow H_{\mathrm{c}}^{q}\left(U,\left.\mathcal{O}_{\lambda}\right|_{U}\right)
$$

induces an isomorphism between the analytic localization of $J(Q, \lambda)_{\min }$ and the sheaf

$$
\left(\left.\mathcal{O}_{\lambda}\right|_{U}\right)^{X}
$$

We can then recover our main result by the Hecht-Taylor equivalence of derived categories.

For the first step of our proof, we continue with the previous notation.

Let

$$
j: \bar{Q} \hookrightarrow X
$$

denote the inclusion and assume $\bar{Q}$ is smooth. We consider the $K$-equivariant sheaf $j^{*}\left(\mathcal{O}_{\lambda}^{\text {alg }}\right)$ defined on $\bar{Q}$. Notice that $j^{*}\left(\mathcal{O}_{\lambda}^{\text {alg }}\right)$ is the sheaf of sections of a $K$-equivariant algebraic line bundle defined on $\bar{Q}$. Let $\mathcal{D}_{\bar{Q}, \lambda}^{\text {alg }}$ denote the sheaf on $\bar{Q}$ 
of (twisted) differential operators of the invertible sheaf $j^{*}\left(\mathcal{O}_{\lambda}^{\text {alg }}\right)$, and let $j+$ be the corresponding direct image functor. Thus,

$$
j+j^{*}\left(\mathcal{O}_{\lambda}^{\mathrm{alg}}\right)
$$

is a $K$-equivariant sheaf of $\mathcal{D}_{\lambda}^{\text {alg }}$-modules.

Proposition 4.2. Suppose $\mathcal{O}_{\lambda}^{\text {alg }}$ is the sheaf of sections of a K-equivariant algebraic line bundle on $X$. Let $Q \subseteq X$ be a K-orbit and suppose $\mathcal{I}(Q, \lambda)$ is the corresponding standard Harish-Chandra sheaf. Assume that the associated variety $\bar{Q}$ is smooth and let $j: \bar{Q} \hookrightarrow X$ be the inclusion. Then there exists a natural isomorphism

$$
j_{+} j^{*}\left(\mathcal{O}_{\lambda}^{\mathrm{alg}}\right) \cong \mathcal{J}(Q, \lambda)
$$

Proof. Let

$$
l: Q \hookrightarrow \bar{Q}
$$

be the inclusion and recall that $i: Q \hookrightarrow X$. Since $Q$ is open in $\bar{Q}$, it is clear that

$$
\left.j^{*}\left(\mathcal{O}_{\lambda}^{\mathrm{alg}}\right)\right|_{Q} \cong i^{*}\left(\mathcal{O}_{\lambda}^{\mathrm{alg}}\right),
$$

as sheaves of $K$-equivariant $\mathcal{D}_{Q}^{\mathrm{alg}}, \lambda$-modules. Furthermore, the direct image $l_{+}$ coincides with the direct image $l_{\bullet}$ in the category of sheaves. By the adjointness property of the direct image

$$
\operatorname{Hom}\left(j^{*}\left(\mathcal{O}_{\lambda}^{\mathrm{alg}}\right), l_{*} i^{*}\left(\mathcal{O}_{\lambda}^{\mathrm{alg}}\right)\right) \cong \operatorname{Hom}\left(\left.j^{*}\left(\mathcal{O}_{\lambda}^{\mathrm{alg}}\right)\right|_{Q}, i^{*}\left(\mathcal{O}_{\lambda}^{\mathrm{alg}}\right)\right)
$$

so the isomorphism above determines a nonzero morphism

$$
j^{*}\left(\mathcal{O}_{\lambda}^{\mathrm{alg}}\right) \rightarrow l_{*} i^{*}\left(\mathcal{O}_{\lambda}^{\mathrm{alg}}\right)
$$

of $K$-equivariant $\mathcal{D} \bar{Q}, \lambda^{\text {alg }}$-modules.

Since $\bar{Q}$ is a closed, smooth subvariety of $X$, Kashiwara's equivalence of categories says that the direct image $j_{+}$establishes an equivalence between the category

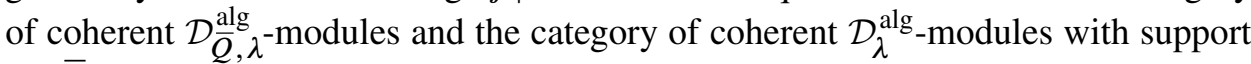
on $\bar{Q}$. Thus, we have a nonzero morphism

$$
j_{+} j^{*}\left(\mathcal{O}_{\lambda}^{\mathrm{alg}}\right) \rightarrow j_{+} l_{*} i^{*}\left(\mathcal{O}_{\lambda}^{\mathrm{alg}}\right) \cong i_{+} i^{*}\left(\mathcal{O}_{\lambda}^{\mathrm{alg}}\right)=\mathcal{I}(Q, \lambda)
$$

this last isomorphism since $i=j \circ l$. Now we simply observe that $j^{*}\left(\mathcal{O}_{\lambda}^{\text {alg }}\right)$ is

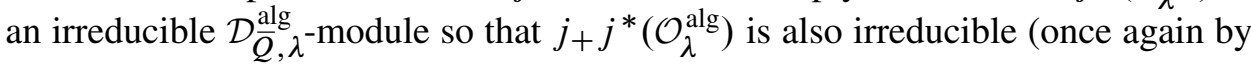
Kashiwara's equivalence). Since the morphism we have defined is nonzero and $i_{+} i^{*}\left(\mathcal{O}_{\lambda}^{\text {alg }}\right)$ has a unique irreducible coherent subsheaf, the proposition is proven. 


\section{Analytic localization and comparison}

We are now ready to introduce the analytic localization. The Hecht-Taylor version of the localization functor is built around the topology of the minimal globalization. One the one hand, Hecht and Taylor consider topological $U_{\Theta}$-modules that have a dual nuclear Fréchet (DNF) topology, where morphisms are continuous morphisms of modules; on the other hand, they define the concept of a DNF sheaf of $\mathcal{D}_{\lambda}$ modules with an accompanying concept of continuous morphisms of DNF sheaves of modules. For $\lambda \in \Theta$, the topological localization

$$
\Delta_{\lambda}^{\mathrm{an}}(M)=\mathcal{D}_{\lambda} \hat{\otimes}_{U_{\Theta}} M
$$

does not have very interesting results, but since free resolutions of DNF modules are complexes of DNF modules, using these sorts of resolutions, one can define a derived functor $L \Delta_{\lambda}^{\text {an }}$. In particular, the analytic localization takes complexes of DNF $U_{\Theta}$-modules to complexes of DNF sheaves of $\mathcal{D}_{\lambda}$-modules (by applying $\Delta_{\lambda}^{\text {an }}$ to the corresponding free resolutions). On the other side of the equation, by using Čech resolutions, Hecht and Taylor show there are enough injectives within the category of DNF sheaves of $\mathcal{D}_{\lambda}$-modules. Thus, by applying the global sections to injective resolutions, one can define a derived global sections functor on complexes of DNF sheaves of $\mathcal{D}_{\lambda}$-modules. The result is then a complex of DNF $U_{\Theta}$-modules. On appropriately defined derived categories, for $\lambda$ regular, it is not hard to show the derived functors $L \Delta_{\lambda}^{\text {an }}$ and $R \Gamma$ are mutual inverses.

In general, one does not know about the homology groups of the analytic localization of a complex of DNF $U_{\Theta}$-modules: these homology groups may very well not be DNF sheaves (although they will be $\mathcal{D}_{\lambda}$-modules). However, the homology groups of the analytic localization of a minimal globalization $M$ (any DNF $U_{\Theta}$-module can be thought of as a complex which is zero in all nonzero degrees) turn out to be DNF sheaves of $\mathcal{D}_{\lambda}$-modules of a very special sort. In order to explain this, we introduce the concept of the geometric fiber of a sheaf of $\mathcal{O}_{X}$-modules. In particular, if $\mathcal{F}$ is a sheaf of $\mathcal{O}_{X}$-modules and $x \in X$, then we define the geometric fiber $T_{x}(\mathcal{F})$ of $\mathcal{F}$ at $x$ by

$$
T_{x}(\mathcal{F})=\mathbb{C} \otimes_{\mathcal{O}_{X, x}} \mathcal{F}_{x},
$$

where $\mathcal{O}_{X, x}$ and $\mathcal{F}_{x}$ denote the corresponding stalks of these sheaves at $x$ and where $\mathcal{O}_{X, x}$ acts on $\mathbb{C}$ by evaluation at $x$. Then, letting $G_{0}[x]$ be the stabilizer of $x$ in $G_{0}$, Hecht and Taylor showed (for $\lambda$ regular) that the geometric fiber

$$
T_{x}\left(L_{p} \Delta_{\lambda}^{\mathrm{an}}(M)\right)
$$

of the $p$-th homology group $L_{p} \Delta_{\lambda}^{\text {an }}(M)$ of the analytic localization of the minimal globalization $M$ is a finite-dimensional (continuous) $\left(\mathfrak{h}_{x}, G_{0}[x]\right)$-module, where 
$\mathfrak{h}_{x}$ acts by the evaluation of $\lambda+\rho \in \mathfrak{h}^{*}$ at $x$, and that the restriction of $L_{p} \Delta_{\lambda}^{\text {an }}(M)$ to the $G_{0}$-orbit $S$ of $x$ is the sheaf of (restricted holomorphic) sections of the corresponding homogeneous vector bundle over $S$.

When $\Theta$ is a regular and $\lambda \in \Theta$ is antidominant, the comparison theorem [Hecht and Taylor 1993] provides a way to understand the analytic localization $L \Delta_{\lambda}^{\text {an }}(M)$ of a minimal globalization $M$, with infinitesimal character $\Theta$, assuming one understands the (derived) geometric fibers of the localization

$$
\Delta_{\lambda}\left(M_{\mathrm{HC}}\right)
$$

of the underlying Harish-Chandra module $M_{\mathrm{HC}}$ of $M$. To explain this result, we introduce the geometric fiber

$$
T_{x}^{\mathrm{alg}}(\mathcal{F})=\mathbb{C} \otimes \mathcal{O}_{X, x}^{\mathrm{alg}} \mathcal{F}_{x}
$$

of a sheaf of $\mathcal{O}_{X}^{\text {alg }}$-modules $\mathcal{F}$ at $x \in X$. Note that $T_{x}^{\text {alg }}$ defines a left exact functor (for example) on the category of quasicoherent $\mathcal{D}_{\lambda}^{\text {alg }}$-modules and that there are corresponding derived functors $L_{p} T_{x}^{\text {alg }}$. When $M_{\mathrm{HC}}$ is a Harish-Chandra module with infinitesimal character $\Theta$ and $\lambda \in \Theta$ is antidominant and regular, Beilinson and Bernstein have shown that the $\mathfrak{h}_{x}$-modules

$$
L_{p} T_{x}^{\mathrm{alg}} \Delta_{\lambda}\left(M_{\mathrm{HC}}\right)
$$

are finite-dimensional (algebraic) $\left(\mathfrak{h}_{x}, K[x]\right)$-modules, where $K[x]$ is the stabilizer of $x$ in $K$ and $\mathfrak{h}_{x}$ acts by the evaluation of $\lambda+\rho \in \mathfrak{h}^{*}$ at $x$. The comparison theorem says that when $x$ is a special point, then there exists a natural equivalence between the finite-dimensional $\left(\mathfrak{h}_{x}, G_{0}[x]\right)$-modules and the finite-dimensional $\left(\mathfrak{h}_{x}, K[x]\right)$-modules (given by the $\left(\mathfrak{h}_{x}, K_{0}[x]\right)$-structure) and that there is a natural isomorphism

$$
L_{p} T_{x}^{\mathrm{alg}} \Delta_{\lambda}\left(M_{\mathrm{HC}}\right) \cong T_{x}\left(L_{p} \Delta_{\lambda}^{\mathrm{an}}(M)\right)
$$

of $\left(\mathfrak{h}_{x}, K_{0}[x]\right)$-modules. Note that a more general comparison theorem, described in exactly these terms is proved in [Bratten 1997, Theorem 7.2].

Theorem 5.1. Suppose $\mathcal{O}_{\lambda}$ is the sheaf of holomorphic sections of a $G_{0}$-equivariant line bundle with regular antidominant parameter $\lambda \in \mathfrak{h}^{*}$. Let $S \subseteq X$ be a $G_{0}$-orbit with vanishing number $q$ and let $U \supseteq S$ be the smallest $G_{0}$-invariant open submanifold that contains $S$. Let $Q$ be the K-orbit that is Matsuki dual to $S$ and suppose the associated variety $\bar{Q}$ is smooth. Then the sheaf cohomology groups

$$
H_{\mathrm{c}}^{p}\left(U,\left.\mathcal{O}_{\lambda}\right|_{U}\right)
$$

vanish except in degree $q$ in which case $H_{\mathrm{c}}^{q}\left(U,\left.\mathcal{O}_{\lambda}\right|_{U}\right)$ is the unique irreducible submodule of the standard module $H_{\mathrm{c}}^{q}\left(S, \mathcal{O}_{\lambda} \mid S\right)$. 
Proof. Utilizing the notation from the previous section, we know that $H_{\mathrm{c}}^{q}\left(S, \mathcal{O}_{\lambda} \mid S\right)$ is the minimal globalization of the standard Beilinson-Bernstein module $I(Q, \lambda)$. Let $J(Q, \lambda) \subseteq I(Q, \lambda)$ be the corresponding unique irreducible Harish-Chandra submodule and $J(Q, \lambda)_{\min }$ its minimal globalization. Consider the complex

$$
R \Gamma\left(\left(\left.\mathcal{O}_{\lambda}\right|_{U}\right)^{X}\right)
$$

of DNF $U_{\Theta}$-modules and let $J(Q, \lambda)_{\min }[-q]$ denote the complex which has zeros in all gradings except $q$, where we have the module $J(Q, \lambda)_{\min }$. The point of our proof is to present a nonzero morphism in the derived category

$$
J(Q, \lambda)_{\min }[-q] \rightarrow R \Gamma\left(\left(\left.\mathcal{O}_{\lambda}\right|_{U}\right)^{X}\right)
$$

such that the induced morphism

$$
L \Delta_{\lambda}^{\mathrm{an}}\left(J(Q, \lambda)_{\min }[-q]\right) \rightarrow L \Delta_{\lambda}^{\mathrm{an}}\left(R \Gamma\left(\left(\left.\mathcal{O}_{\lambda}\right|_{U}\right)^{X}\right)\right) \cong\left(\left.\mathcal{O}_{\lambda}\right|_{U}\right)^{X}[0]
$$

is an isomorphism (we include the place holder [0] to emphasize the fact that we think of the sheaf $\left(\left.\mathcal{O}_{\lambda}\right|_{U}\right)^{X}$ as a complex concentrated in degree zero). By the equivalence of derived categories, we will thus obtain an isomorphism

$$
R \Gamma\left(\left(\left.\mathcal{O}_{\lambda}\right|_{U}\right)^{X}\right) \cong J(Q, \lambda)_{\min }[-q],
$$

which is the desired result.

To present a morphism in the derived category, recall, by Proposition 4.1, that we have a natural inclusion

$$
J(Q, \lambda)_{\min } \rightarrow H_{\mathrm{c}}^{q}\left(U,\left.\mathcal{O}_{\lambda}\right|_{U}\right) .
$$

Since the sheaf cohomology groups of $\left(\left.\mathcal{O}_{\lambda}\right|_{U}\right)^{X}$ vanish in degrees smaller that $q$, a standard truncation argument provides a nonzero morphism in the derived category

$$
H_{\mathrm{c}}^{q}\left(U,\left.\mathcal{O}_{\lambda}\right|_{U}\right)[-q] \rightarrow R \Gamma\left(\left(\left.\mathcal{O}_{\lambda}\right|_{U}\right)^{X}\right) .
$$

Composing this morphism with the inclusion gives the desired result.

We now want to show that the cohomology groups of the complex

$$
L \Delta_{\lambda}^{\mathrm{an}}\left(J(Q, \lambda)_{\min }[-q]\right)
$$

vanish except in degree zero. That is, we want to calculate the homology groups

$$
L_{p} \Delta_{\lambda}^{\mathrm{an}}\left(J(Q, \lambda)_{\min }\right)
$$

and see that they vanish except in degree $q$. To do this, we use the comparison theorem. So we need to calculate the derived geometric fibers of the sheaf

$$
\Delta_{\lambda}(J(Q, \lambda))=\mathcal{J}(Q, \lambda)
$$


Let $j: \bar{Q} \hookrightarrow X$ denote the inclusion. Since $\bar{Q}$ is smooth, we have

$$
\mathcal{J}(Q, \lambda) \cong j_{+} j^{*}\left(\mathcal{O}_{\lambda}^{\mathrm{alg}}\right) .
$$

Thus, calculating the geometric fibers

$$
L_{p} T_{x}^{\mathrm{alg}} \Delta_{\lambda}(J(Q, \lambda)) \cong L_{p} T_{x}^{\mathrm{alg}}\left(j_{+} j^{*}\left(\mathcal{O}_{\lambda}^{\mathrm{alg}}\right)\right)
$$

is a straightforward application of the base change formula for the direct image in the category of TDOs. In particular, since the codimension of $\bar{Q}$ in $X$ is $q$, it follows that, for each $x \in X$,

$$
L_{p} T_{x}^{\text {alg }} \Delta_{\lambda}(J(Q, \lambda))=0 \text { if } p \neq q .
$$

When $x \in \bar{Q}$,

$$
L_{q} T_{x}^{\mathrm{alg}} \Delta_{\lambda}(J(Q, \lambda)) \cong L_{q} T_{x}^{\mathrm{alg}}\left(j_{+} j^{*}\left(\mathcal{O}_{\lambda}^{\mathrm{alg}}\right)\right) \cong T_{x}^{\mathrm{alg}}\left(\mathcal{O}_{\lambda}^{\mathrm{alg}}\right),
$$

and when $x \notin \bar{Q}$,

$$
L_{q} T_{x}^{\mathrm{alg}} \Delta_{\lambda}(J(Q, \lambda))=0 .
$$

In particular, if we let

$$
V=\Gamma\left(X, \mathcal{O}_{\lambda}^{\mathrm{alg}}\right)
$$

be the corresponding irreducible finite-dimensional $(\mathfrak{g}, K)$-module, then for each special point $x \in \bar{Q}$,

$$
L_{q} T_{x}^{\mathrm{alg}} \Delta_{\lambda}(J(Q, \lambda)) \cong V / \mathfrak{n}_{x} V
$$

as $\left(\mathfrak{h}_{x}, K[x]\right)$-modules.

By the comparison theorem, it follows that the homology groups

$$
L_{p} \Delta_{\lambda}^{\mathrm{an}}\left(J(Q, \lambda)_{\min }\right)
$$

vanish except when $p=q$, and this, in turn, implies that the complex

$$
L \Delta_{\lambda}^{\mathrm{an}}\left(J(Q, \lambda)_{\min }[-q]\right)
$$

is quasi-isomorphic to the complex

$$
L_{q} \Delta_{\lambda}^{\mathrm{an}}\left(J(Q, \lambda)_{\min }\right)[0]
$$

which is nonzero only in degree 0 . Hence, the nonzero morphism

$$
L \Delta_{\lambda}^{\mathrm{an}}\left(J(Q, \lambda)_{\min }[-q]\right) \rightarrow\left(\left.\mathcal{O}_{\lambda}\right|_{U}\right)^{X}[0]
$$

in the derived category reduces to a nonzero morphism

$$
L_{q} \Delta_{\lambda}^{\mathrm{an}}\left(J(Q, \lambda)_{\min }\right) \rightarrow\left(\left.\mathcal{O}_{\lambda}\right|_{U}\right)^{X}
$$


of $G_{0}$-equivariant $\mathrm{DNF}$ sheaves of $\mathcal{D}_{\lambda}$-modules. We will prove that this morphism is in fact an isomorphism. In particular, if $O$ is a $G_{0}$-orbit in $U$, then since both $L_{q} \Delta_{\lambda}^{\text {an }}\left(J(Q, \lambda)_{\min }\right) \mid O$ and $\mathcal{O}_{\lambda} \mid O$ are induced equivariant sheaves, it follows that the restricted morphism

$$
\left.\left.L_{q} \Delta_{\lambda}^{\mathrm{an}}\left(J(Q, \lambda)_{\min }\right)\right|_{O} \rightarrow \mathcal{O}_{\lambda}\right|_{O}
$$

is either an isomorphism or zero.

Notice that these limited possibilities for the restricted morphism can also be deduced from the fact that we have a morphism of $\left(\mathcal{D}_{\lambda} \mid O\right)$-modules and both objects are locally free rank one sheaves of $\left(\mathcal{O}_{X} \mid O\right)$-modules. Indeed, if we knew a priori that $L_{q} \Delta_{\lambda}^{\text {an }}\left(J(Q, \lambda)_{\min }\right)$ was a locally free sheaf of $\mathcal{O}_{U}$-modules, then it would follow immediately from standard $\mathcal{D}$-module theory [Hotta et al. 2008, Theorem 1.4.10] that a nonzero morphism of $\mathcal{D}_{\lambda}$-modules would be an isomorphism.

Define $W$ to be the set of $x \in U$ such that the induced morphism

$$
L_{q} \Delta_{\lambda}^{\mathrm{an}}\left(J(Q, \lambda)_{\min }\right)_{x} \rightarrow\left(\mathcal{O}_{\lambda}\right)_{x}
$$

is nonzero. We will show that $W$ is an open set that contains $S$. Since $G_{0}$ acts on $W$ and since $U$ is the smallest $G_{0}$-invariant open set that contains $S$ it will follow from our previous remarks that the morphism in question is an isomorphism.

Consider the composition

$$
L_{q} \Delta_{\lambda}^{\mathrm{an}}\left(J(Q, \lambda)_{\min }\right) \rightarrow\left(\left.\mathcal{O}_{\lambda}\right|_{U}\right)^{X} \rightarrow\left(\left.\mathcal{O}_{\lambda}\right|_{S}\right)^{X},
$$

where the second morphism is the canonical one. Since these morphisms induce the nonzero composition

$$
J(Q, \lambda)_{\min } \rightarrow H_{\mathrm{c}}^{q}\left(U,\left.\mathcal{O}_{\lambda}\right|_{U}\right) \rightarrow H_{\mathrm{c}}^{q}\left(S,\left.\mathcal{O}_{\lambda}\right|_{S}\right),
$$

it follows that the restricted morphism

$$
\left.\left.L_{q} \Delta_{\lambda}^{\mathrm{an}}\left(J(Q, \lambda)_{\min }\right)\right|_{S} \rightarrow \mathcal{O}_{\lambda}\right|_{S}
$$

is an isomorphism and $S \subseteq W$. To show $W$ is open, suppose $x \in W$. Since $\mathcal{O}_{\lambda}$ is a locally free rank one sheaf of $\mathcal{O}_{X}$-modules there is a local section $\sigma$ of $\mathcal{O}_{\lambda}$, defined on a neighborhood of $x$ such that every local section has the form $f \sigma$ where $f$ is a holomorphic function. Since the induced morphism on the geometric fiber

$$
T_{x}\left(L_{q} \Delta_{\lambda}^{\mathrm{an}}\left(J(Q, \lambda)_{\min }\right)\right) \rightarrow T_{x}\left(\mathcal{O}_{\lambda}\right)
$$

is nonzero, it follows that for some open set $W_{1}$ that contains $x$, there is a holomorphic function $f$ defined on $W_{1}$ such that $f(x) \neq 0$, and there is a local section in $\Gamma\left(W_{1}, L_{q} \Delta_{\lambda}^{\text {an }}\left(J(Q, \lambda)_{\min }\right)\right)$ that maps onto $f \sigma$. Thus,

$$
W_{2}=\left\{z \in W_{1}: f(z) \neq 0\right\}
$$


is an open set such that $x \in W_{2} \subseteq W$, and we have finished the proof.

\section{Some additional considerations}

6.1. A tensoring argument. We maintain the notation from the previous section. In particular, $S$ is a $G_{0}$-orbit in $X, Q$ is the $K$-orbit that is Matsuki dual to $S, \mathcal{O}_{\lambda}$ is the sheaf of holomorphic sections of a $G_{0}$-equivariant line bundle on $X$, and so on. When the parameter $\lambda \in \mathfrak{h}^{*}$ is antidominant then it may be the case that the Harish-Chandra module

$$
J(Q, \lambda)=\Gamma(X, \mathcal{J}(Q, \lambda))
$$

is zero. However, when $J(Q, \lambda) \neq 0$, it is the unique irreducible submodule of the standard Beilinson-Bernstein module $I(Q, \lambda)$. When $\lambda$ is antidominant and $J(Q, \lambda) \neq 0$, we will refer to $I(Q, \lambda)$ (as well as its minimal globalization $\left.H_{\mathrm{c}}^{q}\left(S,\left.\mathcal{O}_{\lambda}\right|_{S}\right)\right)$ as a classifying module. Let $U$ be the smallest $G_{0}$-invariant open submanifold that contains $S$. Under the assumption that the associated variety $\bar{Q}$ is smooth and $\lambda$ is antidominant, we can give the following tensoring argument that shows that $H_{\mathrm{c}}^{q}\left(U,\left.\mathcal{O}_{\lambda}\right|_{U}\right)$ is the minimal globalization of $J(Q, \lambda)$. Hence, when $H_{\mathrm{c}}^{q}\left(S,\left.\mathcal{O}_{\lambda}\right|_{S}\right)$ is a classifying module, it follows that $H_{\mathrm{c}}^{q}\left(U,\left.\mathcal{O}_{\lambda}\right|_{U}\right)$ is the unique irreducible submodule.

Lemma 6.1. Assume that $\lambda \in \mathfrak{h}^{*}$ is antidominant, and suppose that the associated variety $\bar{Q}$ is smooth. Then, the sheaf cohomology groups

$$
H_{\mathrm{c}}^{p}\left(U,\left.\mathcal{O}_{\lambda}\right|_{U}\right)
$$

vanish except in degree $q$, in which case $H_{\mathrm{c}}^{q}\left(U,\left.\mathcal{O}_{\lambda}\right|_{U}\right)$ is the minimal globalization of $J(Q, \lambda)$.

Proof. The proof is basically the same as (but simpler than) the proof in [Bratten 1997, Theorem 9.4] with the slight difference that we need to use the description of the irreducible Harish-Chandra sheaf $\mathcal{J}(Q, \lambda)$ from Proposition 4.2 instead of the description for $\mathcal{I}(Q, \lambda)$. We sketch some details to help the reader adapt the notation here to the notation in Section 9 of that reference. From the theory of highest weight modules, one knows there is an irreducible finite-dimensional $G_{0}$-module $F^{\mu}$ which is irreducible as a $\mathfrak{g}$-module and has a highest weight $\mu \in \mathfrak{h}^{*}$ sufficiently dominant that $\lambda-\mu$ is antidominant and regular. Observe that $\mathcal{O}_{\lambda-\mu}$ is the sheaf of holomorphic sections of a $G_{0}$-equivariant line bundle. Let $\Theta$ be the infinitesimal character

$$
\Theta=W \cdot \lambda
$$


If $M$ is a $\mathfrak{g}$-module (or if $\mathcal{M}$ is a sheaf of $\mathfrak{g}$-modules) we let $M_{\Theta}$ (respectively $\mathcal{M}_{\Theta}$ ) denote the corresponding $Z(\mathfrak{g})$-eigenspace. Then, as in the proof of [loc. cit.], we have the natural isomorphisms:

(i) $\left.\left(\left.\mathcal{O}_{\lambda-\mu}\right|_{U} \otimes F^{\mu}\right)_{\Theta} \cong \mathcal{O}_{\lambda}\right|_{U}$, and

(ii) $\left(\mathcal{J}(Q, \lambda-\mu) \otimes F^{\mu}\right)_{\Theta} \cong \mathcal{J}(Q, \lambda)$.

Taking sheaf cohomology, in the first case we obtain

$$
H_{\mathrm{c}}^{p}\left(U,\left.\mathcal{O}_{\lambda}\right|_{U}\right) \cong\left(H_{\mathrm{c}}^{p}\left(U,\left.\mathcal{O}_{\lambda-\mu}\right|_{U}\right) \otimes F^{\mu}\right)_{\Theta},
$$

which implies that the compactly supported sheaf cohomology groups $H_{\mathrm{c}}^{p}\left(U,\left.\mathcal{O}_{\lambda}\right|_{U}\right)$ vanish except when $p=q$, in which case $H_{\mathrm{c}}^{q}\left(U,\left.\mathcal{O}_{\lambda}\right|_{U}\right)$ is the minimal globalization of a Harish-Chandra module. To see which Harish-Chandra module, we begin with the natural isomorphism from the previous section

$$
\left(J_{\lambda-\mu}\right)_{\min } \cong H_{\mathrm{c}}^{q}\left(U,\left.\mathcal{O}_{\lambda-\mu}\right|_{U}\right) .
$$

Therefore, we obtain the isomorphism

$$
\left[\left(J_{\lambda-\mu} \otimes F^{\mu}\right)_{\Theta}\right]_{\min } \cong\left(H_{\mathrm{c}}^{q}\left(U,\left.\mathcal{O}_{\lambda-\mu}\right|_{U}\right) \otimes F^{\mu}\right)_{\Theta} \cong H_{\mathrm{c}}^{q}\left(U,\left.\mathcal{O}_{\lambda}\right|_{U}\right) .
$$

Finally, taking global sections for the isomorphism in (ii), we obtain

$$
\left(J_{\lambda-\mu} \otimes F^{\mu}\right)_{\Theta} \cong J_{\lambda},
$$

which completes the proof of the lemma.

Corollary 6.2. Let $S \subseteq X$ be a $G_{0}$-orbit with vanishing number $q$ and let $U \supseteq S$ be the smallest $G_{0}$-invariant open submanifold that contains $S$. Let $Q$ be the K-orbit that is Matsuki dual to $S$ and suppose the associated variety $\bar{Q}$ is smooth. Let $\mathcal{O}_{\lambda}$ be the sheaf of holomorphic sections of a $G_{0}$-equivariant line bundle and suppose

$$
H_{\mathrm{c}}^{q}\left(S, \mathcal{O}_{\lambda} \mid S\right)
$$

is a classifying module. Then, the sheaf cohomology groups

$$
H_{\mathrm{c}}^{p}\left(U,\left.\mathcal{O}_{\lambda}\right|_{U}\right)
$$

vanish except in degree $q$ in which case $H_{\mathrm{c}}^{q}\left(U,\left.\mathcal{O}_{\lambda}\right|_{U}\right)$ is the unique irreducible submodule of the standard module $H_{\mathrm{c}}^{q}\left(S, \mathcal{O}_{\lambda} \mid S\right)$.

6.2. Maximal parabolic subgroups of complex reductive groups. Suppose $G_{0}$ is a connected, complex reductive group. It turns out that the representation we are studying has a close relationship to the classical parabolic induction when the parabolic subgroup under consideration is maximal. This allowed us to consider some examples (with the help of D. Vogan and A. Paul) to see how the representation works when the associated algebraic variety is singular. In the examples we 
considered, the representation is irreducible only when the associated algebraic variety is nonsingular.

In particular, let $H_{0} \subseteq G_{0}$ be a $\theta$-stable Cartan subgroup ( $\theta$ is the complex conjugation of $G_{0}$ with respect to a compact real form) and let $B_{0} \supseteq H_{0}$ be a Borel subgroup. We consider a maximal, proper parabolic subgroup $P_{0}$ of $G_{0}$ that contains $B_{0}$. These are determined in the following way. Let $W\left(G_{0}\right)$ be the Weyl group of $H_{0}$ (we can think of $W\left(G_{0}\right)$ as the quotient of the normalizer of $H_{0}$ in $G_{0}$ over $\left.H_{0}\right)$. Then, $W\left(G_{0}\right)$ acts naturally on the set of Borel subgroups that contain $H_{0}$. As in the introduction, we let $X_{0}$ be the complex flag manifold of Borel subgroups of $G_{0}$ and let $X_{0}^{\mathrm{c}}$ be the conjugate complex manifold. Then, the flag manifold $X$ of Borel subalgebras of the complexified Lie algebra $\mathfrak{g}$ of $\mathfrak{g}_{0}$ can be identified with the direct product

$$
X=X_{0} \times X_{0}^{\mathrm{c}} .
$$

We have the two actions of $G_{0}$ on $X$ : the diagonal action

$$
g \cdot(x, y)=(g x, g y),
$$

corresponding to the fact that $G_{0}$ is a real group with real Lie algebra $\mathfrak{g}_{0}$, and the action

$$
g \cdot(x, y)=(g x, \theta(g) y),
$$

corresponding to the action of $G_{0}=K$ as the complexification of $K_{0}$. As before let $B_{0}^{\text {op }}$ be the Borel subgroup opposite to $B_{0}$ (this subgroup corresponds to the longest element in $W\left(G_{0}\right)$ ). Then, each $G_{0}$-orbit and each $K$-orbit on $X$ contains exactly one special point of the form

$$
\left(w \cdot B_{0}, B_{0}^{\mathrm{op}}\right) \in X_{0} \times X_{0}^{\mathrm{c}},
$$

so we can identify $G_{0}$-orbits and $K$-orbits with elements of $W\left(G_{0}\right)$. One knows that $Q_{w_{1}} \subseteq \overline{Q_{w_{2}}}$ if and only $w_{1} \preceq w_{2}$ in the Bruhat order $\preceq$ on $W\left(G_{0}\right)$. In particular, the Bruhat interval

$$
[1, w]=\left\{u \in W\left(G_{0}\right): u \preceq w\right\}
$$

characterizes the $K$-orbits $Q_{u}$ contained in $\overline{Q_{w}}$, as well as the $G_{0}$-orbits $S_{u}$ contained in the smallest $G_{0}$-invariant open submanifold $U_{w}$ that contains $S_{w}$. Let $n$ be the number of simple reflections in $W\left(G_{0}\right)$. Observe that the number of $G_{0}$-orbits with vanishing number 1 is exactly $n$. (The closed $G_{0}$-orbit is the unique orbit with vanishing number 0 .)

Let $Y_{0}$ be the generalized complex flag space of $G_{0}$-conjugates to $P_{0}$ and let $Y_{0}^{\mathrm{c}}$ be the complex manifold conjugate to $Y_{0}$. Consider the generalized flag space

$$
Y=Y_{0} \times Y_{0}^{\mathrm{c}},
$$


and let $C$ be the $G_{0}$-orbit of $y=\left(P_{0}, P_{0}\right) \in Y$. Then, $C$ is closed in $Y$, and the $G_{0}$-orbit of $y$ is a real form in $Y$. Let

$$
\pi: X \rightarrow Y
$$

denote the equivariant projection. Then, $\pi^{-1}(C)$ is a closed $G_{0}$-invariant submanifold, and, since $P_{0}$ is a maximal, it contains exactly $n-1$ orbits with vanishing number 1. In particular, if $L_{0} \subseteq P_{0}$ is the Levi factor of $P_{0}$ that contains $H_{0}$ then these $n-1$ orbits correspond to the simple reflections of the Weyl group $W\left(L_{0}\right)$ of $H_{0}$ in $L_{0}$, and the orbits in $\pi^{-1}(C)$ correspond to the elements in $W\left(L_{0}\right)$. Indeed, by intersection with the fiber, these $G_{0}$-orbits give the $L_{0}$-orbits in the complex flag manifold

$$
X_{y}=\pi^{-1}(\{y\})
$$

for $L_{0}$. Let $S$ be the remaining $G_{0}$-orbit with vanishing number 1 and let

$$
U=X-\pi^{-1}(C) \text {. }
$$

Then, $U$ is the smallest $G_{0}$-invariant open set that contains $S$. (To see this fact, since $U$ is open and contains $S$, is sufficient to check that $S$ is the unique $G_{0}$-orbit that is closed in $U$.)

Let $\mathcal{O}_{\lambda}$ be the sheaf of holomorphic sections of a $G_{0}$-equivariant line bundle on $X$ and assume $\lambda$ is antidominant and regular. In a natural way, the sheaf $\mathcal{O}_{\lambda}$ determines a corresponding sheaf of holomorphic sections $\mathcal{O}_{X_{y}, \lambda}$ for an $L_{0}$-equivariant line bundle defined on $X_{y}$. Let

$$
F=\Gamma\left(X_{y}, \mathcal{O}_{X_{y}, \lambda}\right)
$$

be the corresponding irreducible finite-dimensional representation for $L_{0}$ with lowest weight $\lambda+\rho$. In a unique way, this representation extends to an irreducible representation

$$
\omega: P_{0} \rightarrow \mathrm{GL}(F)
$$

Consider the corresponding classical (unnormalized) parabolic induction $I_{P_{0}}^{G_{0}}(F)$, given by

$$
I_{P_{0}}^{G_{0}}(F)=\left\{\text { real analytic functions } \varphi: G_{0} \rightarrow F: \varphi(g p)=\omega\left(p^{-1}\right) \varphi(g)\right\} .
$$

Then, there is a natural isomorphism of $G_{0}$-modules (see, e.g., [Bratten 2008]),

$$
\Gamma\left(\pi^{-1}(C), \mathcal{O}_{\lambda}\right) \cong I_{P_{0}}^{G_{0}}(F) \text { and } H^{p}\left(\pi^{-1}(C), \mathcal{O}_{\lambda}\right)=0 \text { for } p>0,
$$

where we obtain the vanishing by the Leray spectral sequence and the fact that the sheaf cohomology groups of a real analytic vector bundle over a real analytic manifold vanish in positive degree. 
Now, consider the short exact sequence of sheaves

$$
0 \rightarrow\left(\left.\mathcal{O}_{\lambda}\right|_{U}\right)^{X} \rightarrow \mathcal{O}_{\lambda} \rightarrow\left(\left.\mathcal{O}_{\lambda}\right|_{\pi^{-1}(C)}\right)^{X} \rightarrow 0 .
$$

Thus, we have the short exact sequence of representations

$$
0 \rightarrow V \rightarrow I_{P_{0}}^{G_{0}}(F) \rightarrow H_{\mathrm{c}}^{1}\left(U,\left.\mathcal{O}_{\lambda}\right|_{U}\right) \rightarrow 0,
$$

where $V=\Gamma\left(X, \mathcal{O}_{\lambda}\right)$ is the corresponding irreducible finite-dimensional $G_{0}$-module. Therefore, the minimal globalization $H_{\mathrm{c}}^{1}\left(U,\left.\mathcal{O}_{\lambda}\right|_{U}\right)$ is irreducible if and only if the quotient

$$
I_{P_{0}}^{G_{0}}(F) / V
$$

is irreducible.

Let $Q$ be the $K$-orbit Matsuki dual to $S$. Then one would like to know when $\bar{Q}$ is smooth. The calculation for $\operatorname{GL}(n+1, \mathbb{C})$ (which is not difficult) works like this. The Levi factor of $P_{0}$ is characterized by a partition

$$
n_{1}+n_{2}=n+1 \text {, }
$$

where

$$
L_{0}=\operatorname{GL}\left(n_{1}, \mathbb{C}\right) \times \operatorname{GL}\left(n_{2}, \mathbb{C}\right) \subseteq \operatorname{GL}(n+1, \mathbb{C}) .
$$

It turns out that $\bar{Q}$ is smooth if and only $n_{1}$ and $n_{2}$ belong to $\{n, 1\}$. Therefore, $I_{P_{0}}^{G_{0}}(F) / V$ is irreducible in this case. At this point, we contacted D. Vogan to see what was known about the composition factors of these principal series (we asked about the case when $V=\mathbb{C}$ is the trivial $G_{0}$-module). After doing a calculation, he guessed that there are $\min \left\{n_{1}, n_{2}\right\}$ composition factors occurring in the representation $I_{P_{0}}^{G_{0}}(\mathbb{C}) / \mathbb{C}$. Vogan passed this on to Annegret Paul, who confirmed the guess for some low-dimensional examples by using a computer program (apparently the group $\mathrm{GL}(6, \mathbb{C})$ is already a difficult calculation for the algorithms that were used).

Hence, for these examples, the representation $H_{\mathrm{c}}^{1}\left(U,\left.\mathcal{O}_{\lambda}\right|_{U}\right)$ is irreducible if and only if the associated algebraic variety is smooth.

\section{Serre duality}

Since the resolutions used in the Hecht-Taylor construction of the derived category of DNF sheaves of $\mathcal{D}_{\lambda}$-modules are Čech resolutions, perhaps it is worth mentioning that it is not difficult to establish the validity of Serre duality using these sorts of resolutions [Bratten 1997, Section 10]. In particular, let $n$ be the complex dimension of $X$ and let $\Omega^{n}$ be the canonical bundle on $X$. Thus, for $x \in X$, the geometric fiber $T_{x}\left(\Omega^{n}\right)$ of $\Omega^{n}$ at $x$ is given by

$$
T_{x}\left(\Omega^{n}\right)=\bigwedge^{n} \mathfrak{n}_{x}
$$


as a $G_{0}[x]$-module (recall that $G_{0}[x]$ denotes the stabilizer of $x$ in $G_{0}$ ). In particular, $\Omega^{n}$ is a $G_{0}$-equivariant holomorphic line bundle on $X$. Using the unshifted notation from Section 3 of this paper, suppose $\mathcal{O}(\mu)$ is the sheaf of holomorphic sections of a $G_{0}$-equivariant line bundle on $X$. Then, the sheaf of holomorphic sections of the dual bundle is given by $\mathcal{O}(-\mu)$ (i.e., the sheaf of sections of the line bundle associated to the dual geometric fiber). If $U \subseteq X$ is any $G_{0}$-invariant open submanifold of $X$, then Serre duality gives a natural isomorphism of topological $G_{0}$-modules

$$
H_{\mathrm{c}}^{p}\left(U,\left.\mathcal{O}(\mu)\right|_{U}\right)^{\prime} \cong H^{n-p}\left(U,\left.\mathcal{O}(-\mu) \otimes \mathcal{O}\left(\Omega^{n}\right)\right|_{U}\right)
$$

where $H_{c}^{p}\left(U,\left.\mathcal{O}(\mu)\right|_{U}\right)^{\prime}$ denotes the continuous dual of the topological $G_{0}$-module $H_{c}^{p}\left(U,\left.\mathcal{O}(\mu)\right|_{U}\right)$ and $\mathcal{O}\left(\Omega^{n}\right)$ is the sheaf of holomorphic sections of the canonical bundle. In terms of the shifted $\mathcal{D}$-module parameters $\lambda \in \mathfrak{h}^{*}$, we obtain

$$
H_{\mathrm{c}}^{p}\left(U,\left.\mathcal{O}_{\lambda}\right|_{U}\right)^{\prime} \cong H^{n-p}\left(U,\left.\mathcal{O}_{-\lambda}\right|_{U}\right)
$$

for each $p$. In particular, the sheaf cohomology groups of a $G_{0}$-equivariant holomorphic line bundle on a $G_{0}$-invariant open submanifold are maximal globalizations of Harish-Chandra modules.

\section{Acknowledgments}

We would like to take this moment to acknowledge the importance of the work by Hecht and Taylor for these results. We would also like to thank David Vogan and Annegret Paul for being kind enough to help us see how the representation we are studying fails to be irreducible when the associated variety is singular.

\section{References}

[Beilinson and Bernstein 1981] A. Beilinson and J. Bernstein, "Localisation de $g$-modules", C. R. Acad. Sci. Paris Sér. I Math. 292:1 (1981), 15-18. MR 82k:14015 Zbl 0476.14019

[Bott 1957] R. Bott, "Homogeneous vector bundles”, Ann. of Math. (2) 66 (1957), 203-248. MR 19,681d Zbl 0094.35701

[Bratten 1997] T. Bratten, "Realizing representations on generalized flag manifolds", Compositio Math. 106:3 (1997), 283-319. MR 98j:22018 Zbl 0928.22014

[Bratten 1998] T. Bratten, "Finite rank homogeneous holomorphic bundles in flag spaces", pp. 21-34 in Geometry and representation theory of real and p-adic groups (Córdoba, 1995), edited by J. Tirao et al., Progr. Math. 158, Birkhäuser, Boston, 1998. MR 98j:22019 Zbl 0891.22011

[Bratten 2008] T. Bratten, "A geometric embedding for standard analytic modules", Beiträge Algebra Geom. 49:1 (2008), 33-57. MR 2009b:22013 Zbl 1152.22013

[Hecht and Taylor 1990] H. Hecht and J. L. Taylor, "Analytic localization of group representations", Adv. Math. 79:2 (1990), 139-212. MR 91c:22027 Zbl 0701.22005

[Hecht and Taylor 1993] H. Hecht and J. L. Taylor, "A comparison theorem for $\mathfrak{n}$-homology", Compositio Math. 86:2 (1993), 189-207. MR 94c:22015 Zbl 0784.22006 
[Hecht et al. 1987] H. Hecht, D. Miličić, W. Schmid, and J. A. Wolf, "Localization and standard modules for real semisimple Lie groups, I: The duality theorem”, Invent. Math. 90:2 (1987), 297-332. MR 89e:22025 Zbl 0699.22022

[Hotta et al. 2008] R. Hotta, K. Takeuchi, and T. Tanisaki, D-modules, perverse sheaves, and representation theory, Progress in Mathematics 236, Birkhäuser, Boston, 2008. MR 2008k:32022 Zbl 1136.14009

[Matsuki 1979] T. Matsuki, "The orbits of affine symmetric spaces under the action of minimal parabolic subgroups", J. Math. Soc. Japan 31:2 (1979), 331-357. MR 81a:53049 Zbl 0396.53025

[Matsuki 1982] T. Matsuki, "Orbits on affine symmetric spaces under the action of parabolic subgroups”, Hiroshima Math. J. 12:2 (1982), 307-320. MR 83k:53072 Zbl 0495.53049

[Matsuki 1988] T. Matsuki, "Closure relations for orbits on affine symmetric spaces under the action of minimal parabolic subgroups", pp. 541-559 in Representations of Lie groups (Kyoto, Hiroshima, 1986), edited by K. Okamoto and T. Ōshima, Adv. Stud. Pure Math. 14, Academic Press, Boston, 1988. MR 91c:22014 Zbl 0723.22020

[Miličić 1993] D. Miličić, “Algebraic Dิ-modules and representation theory of semisimple Lie groups", pp. 133-168 in The Penrose transform and analytic cohomology in representation theory (South Hadley, MA, 1992), edited by M. Eastwood et al., Contemp. Math. 154, Amer. Math. Soc., Providence, RI, 1993. MR 94i:22035 Zbl 0821.22005

[Schmid 1985] W. Schmid, "Boundary value problems for group invariant differential equations", pp. 311-321 in Élie Cartan et les mathématiques d'aujourd'hui (Lyon, 1984), Astérisque, Société Mathématique de France, Paris, 1985. Numéro Hors Série. MR 87h:22018 Zbl 0621.22014

[Schmid 1989] W. Schmid, "Homogeneous complex manifolds and representations of semisimple Lie groups", pp. 223-286 in Representation theory and harmonic analysis on semisimple Lie groups, edited by P. J. Sally, Jr., Math. Surveys Monogr. 31, Amer. Math. Soc., Providence, RI, 1989. MR 90i:22025 Zbl 0744.22016

[Wolf 1969] J. A. Wolf, "The action of a real semisimple group on a complex flag manifold, I: Orbit structure and holomorphic arc components", Bull. Amer. Math. Soc. 75 (1969), 1121-1237. MR 40 \#4477 Zbl 0183.50901

[Wong 1995] H.-W. Wong, "Dolbeault cohomological realization of Zuckerman modules associated with finite rank representations", J. Funct. Anal. 129:2 (1995), 428-454. MR 96c:22024 Zbl 0855.22014

Received June 27, 2014. Revised March 14, 2015.

José Araujo

FACULTAD DE CIENCIAS EXACTAS

UNICEN

7000 TANDIL

ARGENTINA

araujo@exa.unicen.edu.ar

TIM BRATTEN

FACUltad DE CiEncias Exactas

UNICEN

7000 TANDIL

ARGENTINA

clarkbratten@gmail.com 


\title{
PACIFIC JOURNAL OF MATHEMATICS
}

\author{
msp.org/pjm
}

Founded in 1951 by E. F. Beckenbach (1906-1982) and F. Wolf (1904-1989)

\section{EDITORS}

Don Blasius (Managing Editor)

Department of Mathematics

University of California

Los Angeles, CA 90095-1555

blasius@math.ucla.edu

\author{
Paul Balmer \\ Department of Mathematics \\ University of California \\ Los Angeles, CA 90095-1555 \\ balmer@math.ucla.edu \\ Robert Finn \\ Department of Mathematics \\ Stanford University \\ Stanford, CA 94305-2125 \\ finn@math.stanford.edu \\ Sorin Popa \\ Department of Mathematics \\ University of California \\ Los Angeles, CA 90095-1555 \\ popa@math.ucla.edu
}

\author{
Vyjayanthi Chari \\ Department of Mathematics \\ University of California \\ Riverside, CA 92521-0135 \\ chari@math.ucr.edu \\ Kefeng Liu \\ Department of Mathematics \\ University of California \\ Los Angeles, CA 90095-1555 \\ liu@math.ucla.edu \\ Jie Qing \\ Department of Mathematics \\ University of California \\ Santa Cruz, CA 95064 \\ qing@ cats.ucsc.edu
}

\section{PRODUCTION}

Silvio Levy, Scientific Editor, production@msp.org

\section{SUPPORTING INSTITUTIONS}

ACADEMIA SINICA, TAIPEI

CALIFORNIA INST. OF TECHNOLOGY

INST. DE MATEMÁTICA PURA E APLICADA

KEIO UNIVERSITY

MATH. SCIENCES RESEARCH INSTITUTE

NEW MEXICO STATE UNIV.

OREGON STATE UNIV.

\author{
STANFORD UNIVERSITY \\ UNIV. OF BRITISH COLUMBIA \\ UNIV. OF CALIFORNIA, BERKELEY \\ UNIV. OF CALIFORNIA, DAVIS \\ UNIV. OF CALIFORNIA, LOS ANGELES \\ UNIV. OF CALIFORNIA, RIVERSIDE \\ UNIV. OF CALIFORNIA, SAN DIEGO \\ UNIV. OF CALIF., SANTA BARBARA
}

\author{
Daryl Cooper \\ Department of Mathematics \\ University of California \\ Santa Barbara, CA 93106-3080 \\ cooper@math.ucsb.edu \\ Jiang-Hua Lu \\ Department of Mathematics \\ The University of Hong Kong \\ Pokfulam Rd., Hong Kong \\ jhlu@maths.hku.hk \\ Paul Yang \\ Department of Mathematics \\ Princeton University \\ Princeton NJ 08544-1000 \\ yang@math.princeton.edu
}

These supporting institutions contribute to the cost of publication of this Journal, but they are not owners or publishers and have no responsibility for its contents or policies.

See inside back cover or msp.org/pjm for submission instructions.

The subscription price for 2015 is US \$420/year for the electronic version, and \$570/year for print and electronic.

Subscriptions, requests for back issues and changes of subscribers address should be sent to Pacific Journal of Mathematics, P.O. Box 4163, Berkeley, CA 94704-0163, U.S.A. The Pacific Journal of Mathematics is indexed by Mathematical Reviews, Zentralblatt MATH, PASCAL CNRS Index, Referativnyi Zhurnal, Current Mathematical Publications and Web of Knowledge (Science Citation Index).

The Pacific Journal of Mathematics (ISSN 0030-8730) at the University of California, c/o Department of Mathematics, 798 Evans Hall \#3840, Berkeley, CA 94720-3840, is published twelve times a year. Periodical rate postage paid at Berkeley, CA 94704, and additional mailing offices. POSTMASTER: send address changes to Pacific Journal of Mathematics, P.O. Box 4163, Berkeley, CA 94704-0163.

PJM peer review and production are managed by EditFLOW ${ }^{\circledR}$ from Mathematical Sciences Publishers.

\section{PUBLISHED BY}

\section{mathematical sciences publishers \\ nonprofit scientific publishing}

http://msp.org/

(C) 2015 Mathematical Sciences Publishers 


\section{PACIFIC JOURNAL OF MATHEMATICS}

Volume $277 \quad$ No. $2 \quad$ October 2015

The Borel-Weil theorem for reductive Lie groups

JosÉ ARAUJO and TIM BRATTEN

A curvature flow unifying symplectic curvature flow and pluriclosed

287 flow

SONG DAI

Representations of knot groups into $\mathrm{SL}_{n}(\mathbb{C})$ and twisted Alexander polynomials

Michael HeUsener and JoAn PORTi

Approximations by maximal Cohen-Macaulay modules

HENRIK HOLM

Patterson-Sullivan currents, generic stretching factors and the asymmetric Lipschitz metric for outer space

ILYA KAPOVICH and MARTIN LUSTIG

On recurrence over subsets and weak mixing

JiAn Li, Piotr Oprocha and GUOHUA Zhang

Représentations de Steinberg modulo $p$ pour un groupe réductif sur un 425 corps local

TONY LY

Calculating two-strand jellyfish relations

DAVID PENNEYS and EMILY PETERS 